\title{
$\mathrm{CO}_{2}$ 均相催化氢化研究进展
}

\author{
李 勇 $a$ 王 征 ${ }^{b, c}$ 刘庆涁 $*, a$ \\ ( ${ }^{a}$ 河北师范大学化学与材料科学学院 石家庄 050024) \\ ( ${ }^{b}$ 中国科学院化学研究所 工程塑料重点实验室 北京 100190) \\ ( ${ }^{c}$ 中国科学院大学 北京 100049)
}

\begin{abstract}
摘要 由于近年来可持续发展战略的兴起和对全球变暖问题关注，二氧化碳 $\left(\mathrm{CO}_{2}\right)$ 作为资源用于化学合成已经引起越 来越多化学家的兴趣. 而在利用 $\mathrm{CO}_{2}$ 的研究中, 催化氢化 $\mathrm{CO}_{2}$ 是 $\mathrm{CO}_{2}$ 资源利用的重要手段之一, 均相催化反应具有反 应条件温和、活性高及催化体系易于调控等优点, 使得 $\mathrm{CO}_{2}$ 均相催化氢化尤其是催化生产甲酸、甲醛、甲醇以及胺类 化合物衍生物等方面成为当前 $\mathrm{CO}_{2}$ 资源合理利用的重要课题, 具有广阔的应用前景. 对近年来金属有机络合物催化的 均相氢化 $\mathrm{CO}_{2}$ 的研究进展进行了综述, 主要介绍了催化生产甲酸、甲醛、甲醇以及作为 $\mathrm{C}_{1}$ 合成子方面中的均相催化剂 的种类与结构、活性与选择性以及均相催化氢化 $\mathrm{CO}_{2}$ 的反应机理等方面的研究.
\end{abstract}

关键词 均相催化氢化; $\mathrm{CO}_{2} ;$ 金属有机络合物

\section{Progress in Homogeneous Catalytic Hydrogenation of $\mathrm{CO}_{2}$}

\author{
Li, Yong ${ }^{a} \quad$ Wang, Zheng ${ }^{b, c} \quad$ Liu, Qingbin ${ }^{*, a}$ \\ $\left({ }^{a}\right.$ College of Chemistry and Material Science, Hebei Normal University, Shijiazhuang 050024) \\ ( ${ }^{b}$ Key Laboratory of Engineering Plastics and Beijing National Laboratory for Molecular Science, \\ Institute of Chemistry, Chinese Academy of Sciences, Beijing 100190) \\ $\left({ }^{c}\right.$ University of Chinese Academy of Sciences, Beijing 100049)
}

\begin{abstract}
In recent years, due to the rising of sustainable development strategies and concerns about global warming, chemists have pay more attention to the application of carbon dioxide $\left(\mathrm{CO}_{2}\right)$ as a resource for chemical synthesis. Thereinto, in the research of the using of $\mathrm{CO}_{2}$, catalytic hydrogenation of $\mathrm{CO}_{2}$ is one of the momentous means for the utilization of $\mathrm{CO}_{2}$. The homogeneous catalytic reaction has the advantage of mild reaction condition, high activity and easy regulation of the catalytic system and so forth, so the homogeneous catalytic hydrogenation $\mathrm{CO}_{2}$ becomes to be a hot topic in utilization of $\mathrm{CO}_{2}$ resource, especially in the catalytic production of formic acid, formaldehyde, methanol and amine derivatives, it has extensive application prospects. In this paper, the recent progress in the research of homogeneously hydrogenated $\mathrm{CO}_{2}$ catalyzed by organometallic complexes is reviewed. It included that types and structures, activity and selectivity of homogeneous catalysts for the catalytic production of formic acid, formaldehyde, mathanol and as $\mathrm{C}_{1}$ synthons, and the advance in the reaction mechanism of homogeneous catalytic hydrogenation of $\mathrm{CO}_{2}$.

Keywords homogeneous catalytic hydrogenation; carbon dioxide; organometallic complexes
\end{abstract}

在全球大规模工业化生产的背景下，随着人类日益 增长的能源需求以及不可再生的化石能源的不断消耗, 每年人为的 $\mathrm{CO}_{2}$ 排放量巨大, $\mathrm{CO}_{2}$ 过度排放造成温室效应 加剧, 气候反常, 迫使人们不得不关注 $\mathrm{CO}_{2}$ 的再利用等 问题 ${ }^{[1]}$. 为了实现全球温室气体减排目标, 除了增加可再 生能源技术的使用和改进效率, 碳捕获和储存也将成为
未来减少二氧化碳的重要手段. 然而, 即使通过广泛实 施可再生能源发电来摆脱煤炭发电，但是来自水泥、钢铁 和化工等产业产生的大量 $\mathrm{CO}_{2}$ 仍然会产生排放. 因此采 用 $\mathrm{CO}_{2}$ 作为碳源, 合成各种有机及高分子产品, 即可减 少 $\mathrm{CO}_{2}$ 排放, 又可以生产高附加值的产品满足人类的需 求. 成为全球各国政府与科学界研究的重要课题 ${ }^{[2]}$.

\footnotetext{
* Corresponding author. E-mail: qbinliu@yahoo.com Received February 25, 2017; revised March 30, 2017; published online April 21, 2017.
}

Project supported by the National Natural Science Foundation of China (No. 21476060) and the Natural Science Foundation of Hebei Province (No. B2014205049)

国家自然科学基金(No. 21476060)及河北省自然科学基金(No. B2014205049)资助项目. 
当今国际上对 $\mathrm{CO}_{2}$ 这一储量丰富的原料的资源化 利用, 除植物光合作用之外, 化学法是人工利用 $\mathrm{CO}_{2}$ 最 为主要的方法, 根据分子转化类型分为: (1) $\mathrm{CO}_{2}$ 未还原, 生成有机产物 ${ }^{[1 \sim 3]}$ (如合成尿素、水杨酸、碳酸酯、环碳 酸酯, 聚碳酸酯类等); (2) $\mathrm{CO}_{2}$ 完全被还原成甲烷或饱 和烃 $[1,2,3 \mathrm{a}, 3 \mathrm{k}, 4]$ (成为燃料能源); (3) 部分还原并参与合成多 种官能团化合物 ${ }^{[1,2,3 a, 3 k, 5]}$ (图 1). 在众多 $\mathrm{CO}_{2}$ 资源利用的 研究方法中, 其中催化氢化 $\mathrm{CO}_{2}$ 的方法是最具吸引力且 应用前景符合 “绿色化学”理念的方法 ${ }^{[1 b, 2]}$. 催化氢化 $\mathrm{CO}_{2}$ 是指在一定条件下, 在催化剂的催化作用下, 使 $\mathrm{CO}_{2}$ 和 $\mathrm{H}_{2}$ (或者其他供氢体)发生还原反应生成甲酸、甲 醛、甲醇等有机化合物的方法 ${ }^{[1]}$. 下面将从均相催化氢 化 $\mathrm{CO}_{2}$ 成甲酸及衍生物、甲醛、甲醇及作为 $\mathrm{C}_{1}$ 合成子 四部分综述 $\mathrm{CO}_{2}$ 催化氢化的研究进展.

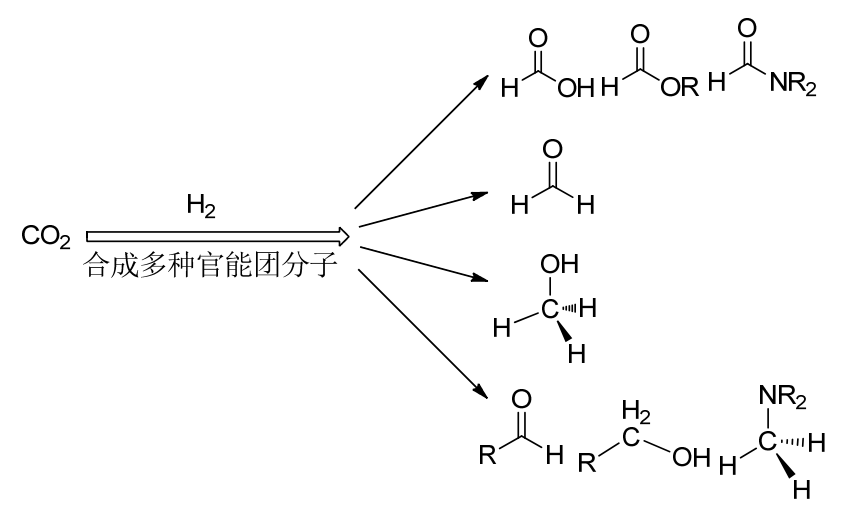

图 1 利用二氧化碳作为碳源的氢化反应研究路线的说明性 实例

Figure1 Illustrative examples of the utilization of carbon dioxide as a carbon source by hydrogenation

\section{1 催化氢化 $\mathrm{CO}_{2}$ 成甲酸及衍生物}

甲酸是在 1670 年从白蚁和红蚂蚁的毒液中发现的. 甲酸作为储氢载体, $\mathrm{H}_{2}$ 存储容量为甲酸分子量的 $4.4 \%$, 甲酸常温为液体, 便于运输过程中的存储和取用, 与其 他储氢技术相比具有较大的优势. 另外甲酸是一种完全 可生物降解的且毒性较低的有机化合物 ${ }^{[6]}$, 其在皮革鞣 制、纺织和橡胶行业具有广阔的应用市场. 自 2006 年初 禁用抗生素作为生长促进剂以后, 甲酸在青咜助剂和动 物饲料中的使用也在逐年上升, 而自欧盟成立以来, 欧 洲成为了该应用的最大消费市场 ${ }^{[3 \mathrm{a}]}$. 甲酸的年产量已经 超过了 60 万吨, 其中 BASF 是甲酸最大的生产商, BASF 生产甲酸的成熟工艺是以甲醇和 $\mathrm{CO}$ 为原料, 经过间接 羰基化、水解生成甲酸(Scheme 1). 而难题在于甲酸的 分离提纯造成的生产成本及能耗过大 ${ }^{[6]}$. 因此开发新型 催化剂氢化 $\mathrm{CO}_{2}$ 成甲酸以降低生产成本和能源消耗是 一个极具现实意义的课题.

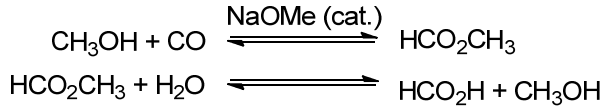

图式 1 目前主要通过 $\mathrm{CO}$ 和水解甲酸甲酯生产甲酸的方法 Scheme 1 Current main process for the production of formic acid from $\mathrm{CO}$ and water via methyl formate

\section{1 将 $\mathrm{CO}_{2}$ 氢化成甲酸和甲酸盐}

1935 年由 Farlow 和 Adkins ${ }^{[7]}$ 报道了首例雷尼镍作 为催化剂催化氢化 $\mathrm{CO}_{2}$ 成甲酸. 之后很少有非均相催化 剂被报道. 其中 2011 年报道的非均相钉金属负载 $\mathrm{Al}_{2} \mathrm{O}_{3}$ 催化剂在 $80{ }^{\circ} \mathrm{C}$ 时显示出对 $\mathrm{CO}_{2}$ 的活性, TON 值达到 $120^{[8]}$. 一般来说, 由于非均相催化剂的活性仍然有限, 而均相催化剂通过配体的设计在催化 $\mathrm{CO}_{2}$ 表现出更好 的性能. 近年来, 均相催化氢化 $\mathrm{CO}_{2}$ 成甲酸的报道很 多 ${ }^{[1,9]}$, 本文将重点介绍代表性的均相催化剂.

1976 年, Inoue 等 ${ }^{[10]}$ 报道了首例用于二氧化碳氢化 成甲酸的均相催化剂. 其报道了 $\mathrm{Pd}, \mathrm{Ni}, \mathrm{Rh}, \mathrm{Ru}$ 和 $\mathrm{Ir}$ 的金 属络合物, 并测试了单膦和双膦配体催化 $\mathrm{CO}_{2}$ 的活性. 认为 “第 VIII 族的大多数过渡金属络合物都可作为催化 氢化 $\mathrm{CO}_{2}$ 成甲酸的催化剂”. 其中 $\left[\mathrm{Ru}(\mathrm{H})_{2}\left(\mathrm{PPh}_{3}\right)_{4}\right]$ 的催 化活性最高, 在温和条件下 $\left(25{ }^{\circ} \mathrm{C}, 2.5 \times 10^{6} \mathrm{~Pa} \mathrm{CO}_{2}\right.$, $2.5 \times 10^{6} \mathrm{~Pa} \mathrm{H}_{2}$ ) 和 $\mathrm{NEt}_{3}$ 存在下 TON 值达到 87. 1992 年, Leitner 等 ${ }^{[11]}$ 报道了使用由 $\left[\left\{\mathrm{Rh}(\mathrm{cod}) \mathrm{Cl}_{\}_{2}}\right](\mathrm{cod}=\right.$ 环辛二 烯)和双齿配体 1,4-双(二苯基膦基)-丁烷(dppb)合成的 催化剂, 在 DMSO 中和 $\mathrm{NEt}_{3}$ 存在下以及温和反应条件 下 $\left(25{ }^{\circ} \mathrm{C}, 2.0 \times 10^{6} \mathrm{~Pa} \mathrm{CO}_{2}, 2.0 \times 10^{6} \mathrm{~Pa} \mathrm{H}_{2}\right) \mathrm{TON}$ 值达到 1150 , TOF 值为 $52 \mathrm{~h}^{-1}$.

1993 年, Leitner 课题组 ${ }^{[12]}$ 报道了首例在水性介质中 有效催化氢化 $\mathrm{CO}_{2}$ 的案例. 其选用 $\left[\mathrm{RhCl}(\mathrm{TPPTS})_{3}\right]$ (TPPTS $=$ 三邻磺酸钠苯基膦)作催化剂, 在二甲胺水溶 液及温和条件 $\left(25{ }^{\circ} \mathrm{C}, 2.0 \times 10^{6} \mathrm{~Pa} \mathrm{CO}_{2}, 2.0 \times 10^{6} \mathrm{~Pa} \mathrm{H}_{2}\right)$ 下 TON 值达到 3439, TOF 值为 287 , 且并没有 $N, N$-二甲 酰胺(DMF)生成. 该催化剂在 $81{ }^{\circ} \mathrm{C}$ 下反应, TOF 值达到 了 $7260 \mathrm{~h}^{-1} .1994$ 年, Noyori 课题组 ${ }^{[13]}$ 引入了超临界 $\mathrm{CO}_{2}$ $\left(\mathrm{scCO}_{2}\right)$ 作为氢化 $\mathrm{CO}_{2}$ 成甲酸的反应介质. 通过使用在 $\mathrm{scCO}_{2}$ 中可溶的催化剂 $\left[\mathrm{RuH}_{2}\left(\mathrm{PMe}_{3}\right)_{4}\right]$ 和 $\left[\mathrm{RuCl}_{2}-\right.$ $\left(\mathrm{PMe}_{3}\right)_{4}$ ](图 2), 在 $2.05 \times 10^{7} \mathrm{~Pa}$ 和 $50{ }^{\circ} \mathrm{C}$ 条件下实现高达 7200 的 TON 和 $1400 \mathrm{~h}^{-1}$ 的 TOF.
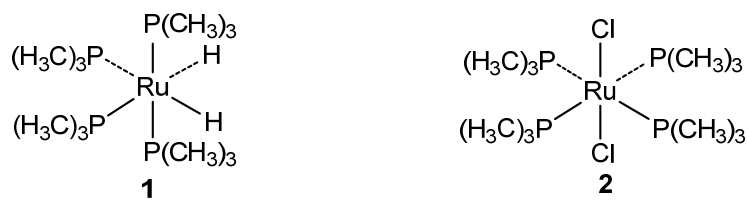

图 2 在超临界 $\mathrm{CO}_{2}$ 中钉金属催化氢化 $\mathrm{CO}_{2}$ 生成甲酸 Figure 2 Catalytic hydrogenation of $\mathrm{CO}_{2}$ to formic acid by ruthenium in supercritical $\mathrm{CO}_{2}\left(\mathrm{scCO}_{2}\right)$ 
2004 年, Himeda 等 ${ }^{[14 a]}$ 报道了以 $\mathrm{Cp}^{*}\left(\mathrm{Cp}^{*}=\right.$ 1,2,3,4,5-五甲基环戍二烯基)为配体合成的半夹心型络 合物氢化 $\mathrm{KOH}$ 水溶液中 $\mathrm{CO}_{2}$ 的案例. 在 $120{ }^{\circ} \mathrm{C}$ 和 $6.0 \times 10^{6} \mathrm{~Pa} \mathrm{H}_{2}$ 条件下, 以 $[\mathrm{IrCp} * \mathrm{Cl}(\mathrm{dhpt})] \mathrm{Cl}(\mathrm{dhpt}=4,7-$ 二羟基-1,10-菲咯啉)作催化剂(Scheme 2, 3, 4), TOF 值达 到 $2.3 \times 10^{4} \mathrm{~h}^{-1} .2007$ 年, 他们用相同的催化剂通过优化 条件 TON 达到 $1.9 \times 10^{5}$, TOF 达到 $4.2 \times 10^{4} \mathrm{~h}^{-1}$. 且通过 使用轻微改性的络合物 $[\mathrm{IrCp} * \mathrm{Cl}(4 \mathrm{dhbp})] \mathrm{Cl}(\mathbf{5}, 4 \mathrm{dhbp}=$ 4,4'-二羟基-2,2'-联吡啶)作为催化剂, 使 TON 达到 $2.22 \times 10^{5}$, TOF 达到 $3.3 \times 10^{4} \mathrm{~h}^{-1[14 b]}$.

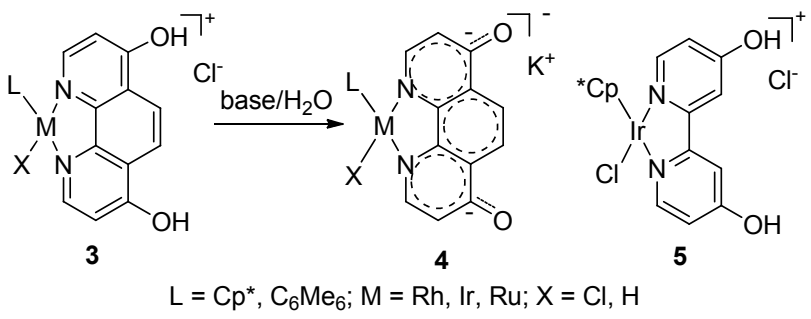

图式 $2 \mathrm{Cp}$ *和 $\mathrm{N}$-供体为配体合成的半夹心型络合物

Scheme 2 Synthesized half-sandwich complexes with $\mathrm{Cp}^{*}$ and $\mathrm{N}$-donor as ligands

2009 年, Nozaki 课题组 ${ }^{[15]}$ 报道了使用 $\operatorname{Ir}(\mathrm{III})$-PNP 螯 型络合物氢化 $\mathrm{KOH}$ 水溶液中的 $\mathrm{CO}_{2}$. 用 $\left[\mathrm{IrH}_{3}(\mathrm{PNP})\right]$ $\left(\mathrm{PNP}=\mathrm{CH}_{2} \mathrm{P}^{i} \mathrm{Pr}_{2}\right)_{2} \mathrm{C}_{5} \mathrm{H}_{3} \mathrm{~N}$ ) 作为催化剂(图 3, 6) 在反应温 度为 $200{ }^{\circ} \mathrm{C}$ 时, TOF 达到 $1.5 \times 10^{5} \mathrm{~h}^{-1}$; 并且在 $120{ }^{\circ} \mathrm{C}$ 下 反应 $48 \mathrm{~h}$ 后 TON 达到 $3.5 \times 10^{6}$, 其对应甲酸盐的浓度 约为 $0.7 \mathrm{~mol} / \mathrm{L} .2011$ 年, Peris 等 ${ }^{[16]}$ 报道了带有磺酸盐取 代基的 NHC 配体的络合物 $\left[\operatorname{IrI}_{2}(\mathrm{AcO})\right.$ (bis-NHC)](图 3, 7), TON 高达 $1.9 \times 10^{5}$, 其对应甲酸盐的浓度为 $0.4 \mathrm{~mol} \cdot$ $\mathrm{L}^{-1}$. 并且在相似条件下用该络合物以 $i-\mathrm{PrOH}$ 作氢供体, 氢化 $\mathrm{CO}_{2}$ 的 TON 达到 2700. 最近, Pidko 及其同事 ${ }^{[17]}$ 报 道了用 Ru-PNP 型络合物 $[\mathrm{RuCl}(\mathrm{H})(\mathrm{CO})(\mathrm{PNP})](\mathrm{PNP}=$ $\left.\left(2,6-\mathrm{CH}_{2} \mathrm{P}^{t} \mathrm{Bu}_{2}\right)_{2} \mathrm{C}_{5} \mathrm{H}_{3} \mathrm{~N}\right)\left(\mathbf{8}\right.$, 图 3), 在在 $1 \times 10^{6} \mathrm{~Pa} \mathrm{H}_{2}, 3 \times$ $10^{6} \mathrm{~Pa} \mathrm{CO}_{2}$ 压力, $120{ }^{\circ} \mathrm{C}$ 下下 $\mathrm{TOF}$ 高达 $1.1 \times 10^{6} \mathrm{~h}^{-1}$ 其中 对应甲酸盐浓度约 $1.2 \mathrm{~mol} \cdot \mathrm{L}^{-1}$. 在总压只有 $5 \times 10^{5} \mathrm{~Pa}$ 反应温度为 $90{ }^{\circ} \mathrm{C}$ 时

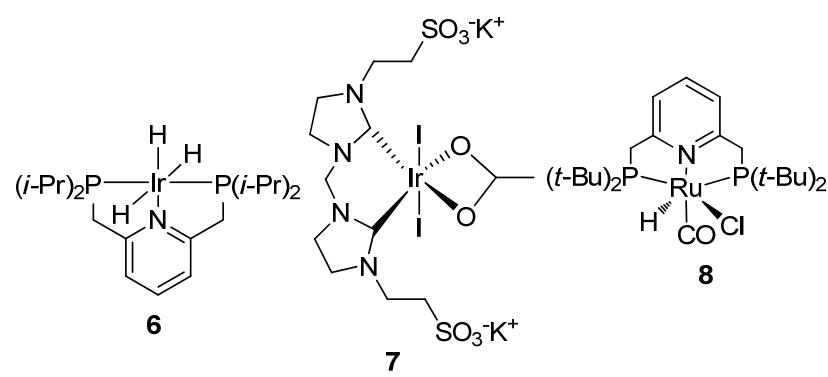

图 3 近年来报道的催化氢化 $\mathrm{CO}_{2}$ 生成甲酸的金属催化剂

Figure 3 Metal catalysts for catalytic hydrogenation of $\mathrm{CO}_{2}$ to formic acid in recent years
TOF 仍然达到 $6.0 \times 10^{4} \mathrm{~h}^{-1}$, 而在 $65{ }^{\circ} \mathrm{C}$ 条件下显著降低 至 $7900 \mathrm{~h}^{-1}$.

\section{2 廉价金属催化剂催化氢化 $\mathrm{CO}_{2}$ 成甲酸}

贵金属催化剂已经成功地氢化 $\mathrm{CO}_{2}$ 合成甲酸及甲 酸盐，因此可以使用类似生产乙酸或加氢甲酰化生产工 艺进行大规模生产，即使用贵金属催化剂，采用高效分 离产物，使金属催化剂循环再利用，实现连续或准连续 操作方法也可进行工业化应用，另外通过开发廉价和储 量丰富的非贵金属催化剂降低用于氢化 $\mathrm{CO}_{2}$ 生产成本 也十分必要.

2003 年, Jessop 等 ${ }^{[18]}$ 使用高压组合篮选法确定金属 的催化活性，这些金属包括 $\mathrm{Co}, \mathrm{Cr}, \mathrm{Fe}, \mathrm{In}, \mathrm{Mo}, \mathrm{Nb}$ 和 $\mathrm{Ni}$, 1,8-二氮杂双环 [5.4.0]十一碳-7-烯(DBU)作为碱, 在 1.0 $\times 10^{7} \mathrm{~Pa}$ 总压力和 $50{ }^{\circ} \mathrm{C}$ 反应条件下活性最高的组合为 $\mathrm{NiCl}_{2} / \mathrm{dcpe}\left(\mathrm{dcpe}=\mathrm{Cy}_{2} \mathrm{PCH}_{2} \mathrm{CH}_{2} \mathrm{PCy}_{2}, \mathrm{TON}=117, \mathrm{TOF}=\right.$ $\left.16 \mathrm{~h}^{-1}\right), \mathrm{FeCl}_{3} / \mathrm{dcpe}\left(\mathrm{TON}=113, \mathrm{TOF}=15 \mathrm{~h}^{-1}\right)$ 和 $\mathrm{MoCl}_{3} / \mathrm{dcpe}\left(\mathrm{TON}=63, \mathrm{TOF}=8 \mathrm{~h}^{-1}\right)$, 在 $\mathrm{H}_{2}$ 和 $\mathrm{CO}_{2}$ 总压 力为 $2 \times 10^{7} \mathrm{~Pa}$ 时使用 $\left[\mathrm{NiCl}_{2}\right.$ (dcpe) $] \mathrm{TON}$ 为 4400.2010 年, Beller 等 ${ }^{[19 a}$ 报道了铁系催化剂 $\mathrm{Fe}\left(\mathrm{BF}_{4}\right)_{2} / \mathrm{PP}_{3}\left[\mathrm{PP}_{3}=\right.$ $\mathrm{P}\left(\mathrm{CH}_{2} \mathrm{CH}_{2} \mathrm{PPh}_{2}\right)_{3}$ 将碳酸氢钠氢化成甲酸钠, $\mathrm{TON}$ 为 660 , 其转化率为 $88 \%\left(80{ }^{\circ} \mathrm{C}, 6.0 \times 10^{6} \mathrm{~Pa} \mathrm{H}_{2}\right)$. 通过使用四齿 配体三 [(2-二苯基膦基)苯基 $]\left(\mathrm{P}_{4}\right)$ 和空气中稳定的催化剂 $\left[\mathrm{FeF}\left(\mathrm{P}_{4}\right)\right]\left[\mathrm{BF}_{4}\right]$ 氢化甲醇溶液中的碳酸氢 盐 ${ }^{[19 b]}$, 在 $100{ }^{\circ} \mathrm{C}$ 和 $6.0 \times 10^{6} \mathrm{~Pa} \mathrm{H}_{2}$ 条件下 TON 达 7546(产率 77\%), TOF 为 $377 \mathrm{~h}^{-1}$. 该催化剂也能在甲醇溶液中催化氢化 $\mathrm{CO}_{2}$ 生成甲酸、甲酸甲酯和甲酰胺. 也可在水和 $\mathrm{NEt}_{3}$ 存 在下在甲醇溶液中氢化 $\mathrm{CO}_{2}$ 成甲酸, $100{ }^{\circ} \mathrm{C}$, 总压力 $6.0 \times 10^{6} \mathrm{~Pa}$ 反应 $20 \mathrm{~h}$ 后 $\mathrm{TON}$ 为 1897 . 由此看出, 多齿 膦配体铁催化剂活性较高.

2011 年, Milstein 等 ${ }^{[20]}$ 报道了将 $\mathrm{CO}_{2}$ 氢化成甲酸钠 盐的螯型铁络合物. 在温和的反应条件下 $\left(80{ }^{\circ} \mathrm{C}\right.$, 室温 总压力 $1.0 \times 10^{6} \mathrm{~Pa}$ )使用 $\mathrm{Fe}(\mathrm{II})$ 螯形络合物 $\left[\mathrm{FeH}_{2}(\mathrm{CO})\right.$ $\left(t\right.$-Bu-PNP)] (9, 图 4) 获得 TON 为 788, TOF 为 $156 \mathrm{~h}^{-1}$. 此外，在类似的反应条件下使用相同催化剂和无 $\mathrm{CO}_{2}$ 存 在下氢化碳酸氢钠, TON 为 320 (产率 $32 \%$ ). 2012 年, Beller 等 ${ }^{[21]}$ 报道了钴催化剂 $\mathrm{Co}\left(\mathrm{BF}_{4}\right)_{2} / \mathrm{PP}_{3}\left(\mathrm{PP}_{3}=三[2-(\right.$ 二 苯基膦基)乙基]膦或四磷)显示出与之前报道铁催化剂 体系类似的活性. 分别用乙酰丙酮钴盐 $\left[\mathrm{Co}(\mathrm{acac})_{2}\right]$, 和 $\mathrm{CoCl}_{2}$ 与 $\mathrm{PP}_{3}$ 组合成催化剂, 在 $120{ }^{\circ} \mathrm{C}$ 条件下催化氢化 $\mathrm{CO}_{2}$ 和碳酸氢钠的 TON 高达 3877 , TOF 达到 $194 \mathrm{~h}^{-1}$. 催 化剂也可用于将 $\mathrm{CO}_{2}$ 氢化成甲酸烷基酯和甲酰胺. Muckerman 等 ${ }^{[22]}$ 报道了 $\left[\mathrm{CoCp} *\left(\mathrm{H}_{2} \mathrm{O}\right)(4-\mathrm{dhbp})\right]_{2}{ }^{+}$(4$\mathrm{dhbp}=4,4^{\prime}$-二羟基- $2,2^{\prime}$-联吡啶)类的 $\operatorname{Ir}(\mathrm{III})$ 催化剂(10, 图 4). 在 $100{ }^{\circ} \mathrm{C}$ 和 $8.0 \times 10^{6} \mathrm{~Pa}$ 总压力下将碳酸氢盐水溶液 中 $\mathrm{CO}_{2}$ 氢化, $\mathrm{TOF}$ 为 $39 \mathrm{~h}^{-1}$. 同年, Jeletic 等 ${ }^{[23]}$ 报道了一 
种高活性的钴催化体系, 用 $\left[\mathrm{Co}(\mathrm{dmpe})_{2} \mathrm{H}\right](\mathrm{dmpe}=1,2-$ 双(二甲基膦基)乙烷)(11, 图 4)在胺存在下和温和的反 应条件下 (室温, $2.0 \times 10^{6} \mathrm{~Pa}$ ) 将 THF 溶液中 $\mathrm{CO}_{2}$ 氢化成 甲酸, $\mathrm{TOF}$ 为 $7.4 \times 10^{4} \mathrm{~h}^{-1}$.

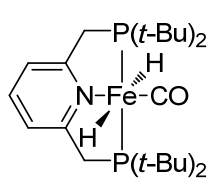

9

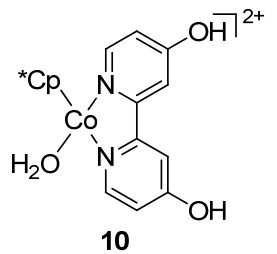

10

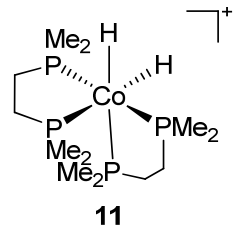

11
图 4 近年来报道的用于氢化 $\mathrm{CO}_{2}$ 生成甲酸的廉价金属催化 剂

Figure 4 Nonprecious metal catalysts for the formation of formic acid by hydrogenation of $\mathrm{CO}_{2}$ reported in recent years

总之, 尽管现在廉价过渡金属催化剂体系的 TON 和 TOF 值通常比最佳的贵金属催化剂体系低两个数量级. 但是通过配体结构的修饰, 有望提高催化剂性能, 并且 这些金属的配位特征与性能的关系有待进一步研究.

\section{3 在无碱条件下氢化 $\mathrm{CO}_{2}$ 成甲酸}

无碱高效氢化 $\mathrm{CO}_{2}$ 成甲酸是十分有吸引力且具有 商业前景的课题. 1989 年, Khan 等 ${ }^{[24]}$ 报道使用络合物 $\mathrm{K}[\mathrm{RuCl}(\mathrm{EDTA}-\mathrm{H})](\mathrm{EDTA}-\mathrm{H}=$ 乙二胺四乙酸盐) 氢化 $\mathrm{CO}_{2}$ 为甲酸和甲醛, 随后分解成水煤气. 在无碱条件和 相对温和的反应条件下 $\left(3 \times 10^{5} \mathrm{~Pa} \mathrm{H}_{2}, 1.7 \times 10^{6} \mathrm{~Pa} \mathrm{CO}_{2}\right.$, $\left.40{ }^{\circ} \mathrm{C}\right)$ 甲酸形成的 TOF 为 $1200 \mathrm{~h}^{-1}$. 然而, 最初形成的 甲酸和甲醛随后分解为 $\mathrm{CO}$ 和 $\mathrm{H}_{2} \mathrm{O}$. 因此, 此体系不能 选择性地形成甲酸. 2004 年, Fukuzumi 等 ${ }^{[25]}$ 报道了首个 $\mathrm{CO}_{2}$ 无碱选择性氢化成甲酸的案例. 用水溶性络合物 $\left[\mathrm{Ru}\left(\eta^{6}-\mathrm{C}_{6} \mathrm{Me}_{6}\right)\left(4,4^{\prime}-\mathrm{OMe}-\mathrm{bpy}\right)\left(\mathrm{H}_{2} \mathrm{O}\right)\right]\left(\mathrm{SO}_{4}\right) \quad\left(4,4^{\prime}-\mathrm{OMe}-\right.$ bpy =二甲氧基-2,2'-联吡啶)(12, 图 5)作催化剂在相对 温和的条件 $\left(5.5 \times 10^{6} \mathrm{~Pa} \mathrm{H}_{2}, 2.5 \times 10^{6} \mathrm{~Pa} \mathrm{CO}_{2}, 40{ }^{\circ} \mathrm{C}\right)$ 下 反应 $70 \mathrm{~h}$ 生成甲酸, TON 达到 55 , 对应甲酸浓度为 0.06

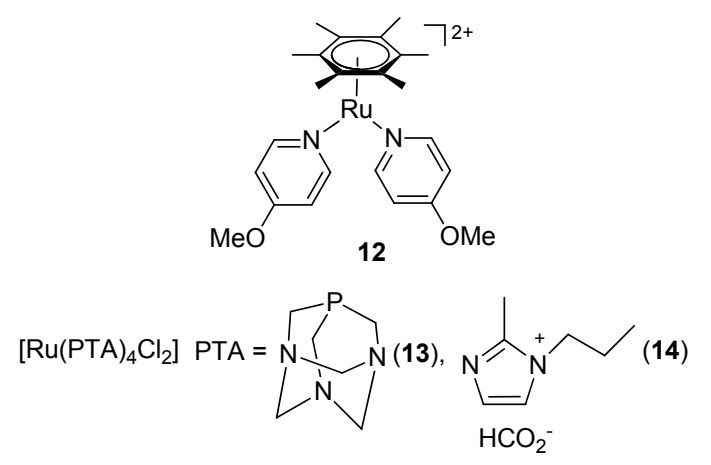

图 5 近年来报道的无碱催化 $\mathrm{CO}_{2}$ 的催化剂 Figure 5 Noalkali-catalyzed $\mathrm{CO}_{2}$-catalyzed catalyst reported in recent years $\mathrm{mol} \cdot \mathrm{L}^{-1}$. 2011 年 Joó 等 ${ }^{[26]}$ 报道了使用络合物 $\left[\mathrm{RhCl}(\mathrm{TPPMS})_{3}\right]$ 作为催化剂时, 在形成甲酸的水溶液中 存在低浓度的甲酸钠, 表明 $\mathrm{HCO}_{2} \mathrm{Na}$ 有可能在生成甲酸 中对催化剂起活化作用.

在 2014 年, Laurenczy 等 ${ }^{[27]}$ 报道了用不同催化剂和 溶剂无碱氢化 $\mathrm{CO}_{2}$ 成甲酸. 用 $\left[\mathrm{RuCl}_{2}(\mathrm{PTA})_{4}\right](\mathrm{PTA}=$ 1,3,5-三氮杂-7-磷杂金刚烷)(13, 图 5)在纯水中和较高 压力下 $\left(1.5 \times 10^{7} \mathrm{~Pa} \mathrm{H}_{2}, 5.0 \times 10^{6} \mathrm{~Pa} \mathrm{CO}_{2}, 60{ }^{\circ} \mathrm{C}\right.$, 室温 $)$ TON 为 159 , 甲酸浓度为 $0.204 \mathrm{~mol} \cdot \mathrm{L}^{-1}$. 通过减压除去 形成的甲酸和溶剂(水)并重新溶解剩余的固体催化剂. 催化剂可重复使用四次而不损失活性.

无碱条件下，甲酸也可以在有机溶剂中形成，通过 溶剂化效应来改变溶液的平衡. 1992 年, Nicholas 等 ${ }^{[28]}$ 报道在四氢呋喃(THF)溶液中将 $\mathrm{CO}_{2}$ 无碱氢化成甲酸. 通过用 $\left[\mathrm{Rh}(\mathrm{nbd})\left(\mathrm{PMe}_{2} \mathrm{Ph}\right)_{3}\right] \mathrm{BF}_{4}(\mathrm{nbd}=$ 降冰片二烯)催化 $\mathrm{CO}_{2}$ 在干燥 $\mathrm{THF}$ 中反应, $\mathrm{TON}$ 为 $34\left(40{ }^{\circ} \mathrm{C}, 4.8 \times 10^{6} \mathrm{~Pa}\right.$ $\mathrm{H}_{2}, 4.8 \times 10^{6} \mathrm{~Pa} \mathrm{CO}_{2}$ ), 对应甲酸浓度为 $0.05 \mathrm{~mol} \cdot \mathrm{L}^{-1}$. 在 相同的条件下使用含有 $0.4 \mathrm{vol} \% \mathrm{H}_{2} \mathrm{O}$ 水的 THF 溶液时, TON 为 78 , 对应甲酸浓度为 $0.11 \mathrm{~mol} \cdot \mathrm{L}^{-1} .2014$ 年, Laurenczy 等 ${ }^{[27]}$ 用 $\left[\mathrm{RuCl}_{2}(\mathrm{PTA})_{4}\right]$ 作为催化剂在无碱条件 下, 在乙醇, 甲醇, 乙腈和碳酸亚丙酯中反应, 在 $50{ }^{\circ} \mathrm{C}$ 和 $1.0 \times 10^{7} \mathrm{~Pa}\left(\mathrm{CO}_{2}: \mathrm{H}_{2}=1: 1\right)$ 条件下反应 $120 \mathrm{~h}$ 后甲 酸浓度为 $0.20 \mathrm{~mol} \cdot \mathrm{L}^{-1}(\mathrm{TON}=72)$. 相同条件下在二甲 亚砜(DMSO)中反应, $120 \mathrm{~min}$ 后 $\mathrm{TON}$ 为 700 , 平均 TOF $=5.8 \mathrm{~h}^{-1}$, 对应甲酸浓度为 $1.93 \mathrm{~mol} \cdot \mathrm{L}^{-1}$. 在 $5.0 \times 10^{6} \mathrm{~Pa}$ 压力下和 $50{ }^{\circ} \mathrm{C}$ 下, 反应 $120 \mathrm{~h}$ 后 TON 为 141 , 平均 TOF 为 $1.2 \mathrm{~h}^{-1}$, 甲酸的浓度仍为 $0.39 \mathrm{~mol} \cdot \mathrm{L}^{-1}$.

2012 年, Leitner 及其同事 ${ }^{[29]}$ 报道了 $\mathrm{CO}_{2}$ 加氢反应在 包含甲酸盐阴离子的 1-丙基-2,3-二甲基咪唑甲酸盐 $\left([\mathrm{PMMIM}]\left[\mathrm{HCO}_{2}\right]\right)(\mathbf{1 4}$, 图 5)中进行时 TON 为 1968, TOF 为 $295 \mathrm{~h}^{-1}$, 对应甲酸浓度为 $3.94 \mathrm{~mol} \cdot \mathrm{L}^{-1}$. 显然, 从生产甲酸工艺上讲, 在无碱条件下氢化 $\mathrm{CO}_{2}$ 成甲酸更 值得深入的研究. 但目前首先应该重点关注对催化剂起 激活作用的碱, 因为这可能是在无碱情况下改善催化剂 性能的关键 ${ }^{[30]}$. 其次是由于无碱体系利用 $\mathrm{HCOOH}$ 与反 应介质的其他强相互作用来补偿不利的熵, 因此不容易 进行分离. 由于反应中存在分解反应, 故产物混合物不 能在催化剂存在下蒸馏, 因此需要特别考虑用于产物分 离和催化剂再循环的替代策略, 固定化催化剂是一种选 择.

固定催化剂是在甲酸蒸馏之前将催化剂分离以避 免蒸馏时甲酸分解的理想方法. 这个方案可以通过将有 机金属催化剂固定在固体载体上或使用多相反应系统 来实现. 2004 年, 郑小明课题组 ${ }^{[1,32]}$ 报道了固定在氨基 官能化二氧化硅上的钓-膦催化剂. 以 $\mathrm{PPh}_{3}$ 作为配体时, 
反应效果最好的体系是 $\mathrm{Si}\left(\mathrm{CH}_{2}\right)_{3} \mathrm{NH}\left(\mathrm{CH}_{2}\right)_{3} \mathrm{CH}_{3} \mathrm{RuCl}_{3}$. 使 用该系统在 $\mathrm{EtOH} / \mathrm{NEt}_{3} / \mathrm{scCO}_{2}\left(80{ }^{\circ} \mathrm{C}, 4.0 \times 10^{6} \mathrm{~Pa} \mathrm{H}_{2}\right.$, $1.2 \times 10^{7} \mathrm{~Pa} \mathrm{CO}_{2}$ ) 条件下 TOF 高达 $1384 \mathrm{~h}^{-1}$. 催化剂可重 复使用两次且活性仅有轻微损失. 热过滤试验表明滤液 没有催化活性，从而证明了催化仅发生在固体材料上的 假设. 2006 年, 他们 ${ }^{[33]}$ 报道了以胺官能团化的聚苯乙烯 为载体材料的 polystyrene- $\mathrm{CH}_{2} \mathrm{NH}\left(\mathrm{CSCH}_{3}-\mathrm{RuCl}_{3}\right) / \mathrm{PPh}_{3}$ 催化剂. 2013 年, Hicks 课题组 ${ }^{[34]}$ 报道了以介孔二氧化硅 为载体的铱催化剂. 在 $\mathrm{H}_{2} \mathrm{O} / \mathrm{NEt}_{3}\left(120{ }^{\circ} \mathrm{C}\right.$, 总压力 $4.0 \times$ $10^{6} \mathrm{~Pa}$, 室温)下 $\mathrm{TOF}$ 为 $1200 \mathrm{~h}^{-1}$. 在 $60{ }^{\circ} \mathrm{C} \mathrm{TOF}$ 仍可达 到 $880 \mathrm{~h}^{-1}$. 催化剂可以通过过滤来回收并可以重复使 用 10 个循环且只有轻微的失活. 在所有这些报道中, 甲 酸/胺加合物的溶液可以经过滤较容易地从催化剂中分 离, 但是不包括这些溶液的进一步后处理. 而这是最耗 费能源的一步. 因此, 开发更好的方法, 整合反应和分 离步骤, 对于 $\mathrm{CO}_{2}$ 转化成甲酸实现工业化应用至关重 要.

\section{4 通过催化氢化 $\mathrm{CO}_{2}$ 合成烷基甲酸酯}

目前工业生产烷基甲酸酯是主要采用两种技术: 羰 基化醇和甲酸酯与醇反应(Scheme 3$)^{[6]}$. 甲酸甲酯大部 分被用作中间体来生产甲酸和甲酰胺. 少部分的甲酸甲 酯用作溶剂、杀虫剂, 或用于固化苯酚-甲酫树脂. 甲酸 甲酯还可用作生产葡萄糖酸甲酯、乙二醇、甲基丙酸酯、 乙醛、乙酸和碳酸二甲酯. 此外, 甲酸甲酯可用于生产 高纯度 $\mathrm{CO}^{[35]}$. 而其他烷基酸甲酯, 例如甲酸乙酯、甲酸 异丁酯、甲酸芐酯和甲酸异戊酯可以用作溶剂、香料和 调味剂.

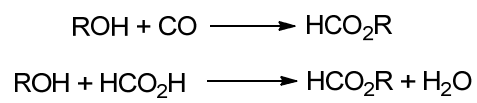

图式 3 工业用于生产甲酸烷基酯的反应 Scheme 3 Industrially used reactions for the production of alkyl formates

1995 年, Jessop 和 Noyori 等 ${ }^{[36]}$ 报道了在甲醇和超临 界 $\mathrm{CO}_{2}$ 下氢化 $\mathrm{CO}_{2}$ 成甲酸甲酯. 使用 $\left[\mathrm{RuCl}_{2}\left(\mathrm{PMe}_{3}\right)_{4}\right]$ 作 为催化剂, 在 $80{ }^{\circ} \mathrm{C}, 8.0 \times 10^{6} \mathrm{~Pa} \mathrm{H}_{2}$ 和 $1.25 \times 10^{7} \mathrm{~Pa} \mathrm{CO}_{2}$ 条件下 TON 为 $3500, \mathrm{TOF}$ 为 $55 \mathrm{~h}^{-1} .1997$ 年, Baiker 课 题组 ${ }^{[37]}$ 使用 $\left[\mathrm{RuCl}_{2}(\mathrm{dppe})_{2}\right.$ ]在类似条件下使得 TOF 高达 $850 \mathrm{~h}^{-1}, \mathrm{TON}$ 高达 12900 . 在 2014 年由 Yadav 等 ${ }^{[38]}$ 报道 了用相同的反应方法捕获 $\mathrm{CO}_{2}$ 并将其氢化成甲酸甲酯 (Scheme 4). $\mathrm{CO}_{2}$ 可以在 $101 \mathrm{kPa}$ 下用 DBU 和甲醇的混 合物有效地捕获, 并在 $101 \mathrm{kPa}$ 和室温下形成离子液体 [DBUH]和碳酸甲酯, 即所谓的 “可转换离子液体”。

在廉价金属有机催化剂方面, 1978 年, Evans 等 ${ }^{[39]}$ 报道了阴离子铁羰基络合物 $\left[\mathrm{Fe}_{3} \mathrm{H}(\mathrm{CO})_{11}\right]^{-}$在相应的醇

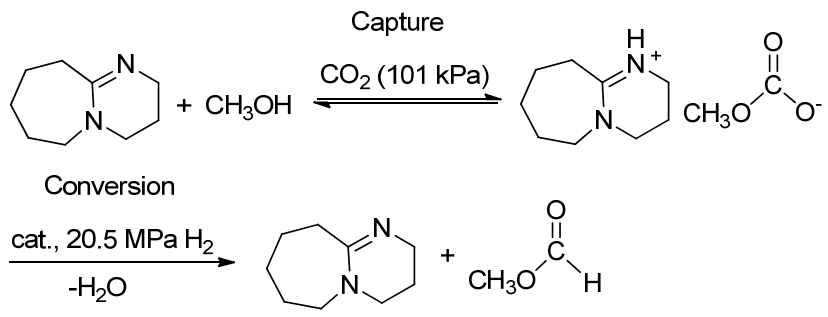

图式 4 捕获 $\mathrm{CO}_{2}$ 并将其氢化成甲酸甲酯的系统

Scheme 4 A system for capturing $\mathrm{CO}_{2}$ and hydrogenating it to methyl formate

存在下催化氢化 $\mathrm{CO}_{2}$ 转化为甲酸甲酯和甲酸乙酯. 在无 碱条件下 $\left(2.0 \times 10^{6} \mathrm{~Pa} \mathrm{CO}_{2}, 2.0 \times 10^{6} \mathrm{~Pa} \mathrm{H}_{2}, 175{ }^{\circ} \mathrm{C}\right)$ 反应 $96 \mathrm{~h}$ 后 TON 为 6.2010 年, Beller 和同事 ${ }^{[40]}$ 表明, 催化剂 $\mathrm{Fe}\left(\mathrm{BF}_{4}\right)_{2} \cdot 6 \mathrm{H}_{2} \mathrm{O} / \mathrm{PP}_{3}\left[\mathrm{PP}_{3}=\mathrm{P}\left(\mathrm{CH}_{2} \mathrm{CH}_{2} \mathrm{PPh}_{2}\right)_{3}\right]$ 和预催化剂 $\left[\mathrm{FeH}\left(\mathrm{H}_{2}\right) \mathrm{PP}_{3}\right]\left[\mathrm{BF}_{4}\right]$ 在相应的醇存在下 $\left(100{ }^{\circ} \mathrm{C}, 6.0 \times 10^{6}\right.$ $\mathrm{Pa} \mathrm{H}_{2}, 3.0 \times 10^{6} \mathrm{~Pa} \mathrm{CO}_{2}$ ) 可有效地催化 $\mathrm{CO}_{2}$ 氢化为甲酸 甲酯 $(T O N=585)$ 、甲酸乙酯 $(T O N=167)$ 和甲酸丙酯 $(\mathrm{TON}=94) .2012$ 年, Beller 课题组 ${ }^{[21]}$ 用类似的 Co 催化 剂 $\mathrm{Co}\left(\mathrm{BF}_{4}\right)_{2} \cdot 6 \mathrm{H}_{2} \mathrm{O} / \mathrm{PP}_{3}$ 或催化剂 $\left[\mathrm{Co}\left(\mathrm{H}_{2}\right) \mathrm{PP}_{3}\right]\left[\mathrm{BPh}_{4}\right]$ 在相 同的反应条件下得到甲酸甲酯的 TON 为 659 , 基于 $\mathrm{NEt}_{3}$ 的产率为 $83 \%$, 产生甲酸乙酯的 TON 为 103 . 同年, 他 们 ${ }^{[196]}$ 报道了使用 $\mathrm{Fe}\left(\mathrm{BF}_{4}\right)_{2} \cdot 6 \mathrm{H}_{2} \mathrm{O}$ 与四齿配体三 $[(2$-二苯 基膦基)甲基]膦得到甲酸甲酯的 TON 为 795. 在甲醇和 $\mathrm{NEt}_{3}$ 的条件下 $\left(100{ }^{\circ} \mathrm{C}, 3.0 \times 10^{6} \mathrm{~Pa} \mathrm{H}_{2}, 3.0 \times 10^{6} \mathrm{~Pa} \mathrm{CO}_{2}\right)$ 的选择性为 $47 \%$.

因为碱的添加通常会产生甲酸加合物和酯的混合 物，因此在无碱条件下进行反应可以简化后处理. 早在 20 世纪 70 年代的研究中已经在使用路易斯酸或根本没 有添加剂, 1983 年 Darensbourg 等 ${ }^{[41]}$ 使用 $\left[\mathrm{Ru}_{4} \mathrm{H}_{3}(\mathrm{CO})_{12}\right]^{-}$ 在无添加剂的情况下获得甲酸甲酯, TON 为 7. 1995 年 Lau 和 $\mathrm{Chen}^{[42]}$ 在乙醇中使用 $\left[\mathrm{Ru}\left(\mathrm{Cl}_{2} \mathrm{bpy}\right)_{2}\left(\mathrm{H}_{2} \mathrm{O}\right)_{2}\right]-$ $\left[\mathrm{CF}_{3} \mathrm{SO}_{3}\right]_{2}$ 在无碱条件下得到甲酸乙酯的 $\mathrm{TON}$ 为 160 , $\mathrm{TOF}$ 为 $20 \mathrm{~h}^{-1} .2011$ 年, Huff 和 Sanford ${ }^{[43]}$ 报道了催化剂 $\left[\mathrm{RuCl}(\mathrm{OAc})\left(\mathrm{PMe}_{3}\right)_{4}\right]$ 在 $135{ }^{\circ} \mathrm{C}, 1.0 \times 10^{6} \mathrm{~Pa} \mathrm{CO}_{2}, 3.0 \times$ $10^{6} \mathrm{~Pa} \mathrm{H}_{2}$ 和没有添加剂且 $\mathrm{NEt}_{3}$ 存在下以 $\mathrm{Sc}(\mathrm{OTf})_{3}$ 作为 路易斯酸条件下, 反应 $16 \mathrm{~h}$ 后 TON 为 $40, \mathrm{TOF}$ 为 32 $\mathrm{h}^{-1}$. 与其他催化剂的组合, 路易斯酸加快了分子醇酯 化过程反应速度, 因此系统性地研究路易斯酸作为助催 化剂使 $\mathrm{CO}_{2}$ 无碱氢化成甲酸酯对由 $\mathrm{CO}_{2}$ 合成甲酸酯具 有重要意义.

\section{5 通过催化氢化 $\mathrm{CO}_{2}$ 合成甲酰胺}

甲酰胺可以通过 $\mathrm{CO}_{2}$ 与一种或多种含 $\mathrm{N}-\mathrm{H}$ 官能团 的胺反应而得到. 该机理包括将 $\mathrm{CO}_{2}$ 氢化成甲酸铵, 然 后脱水缩合成甲酰胺(Scheme 5$)^{[44,45]}$. 甲酰胺和二甲基 甲酰胺的制备具有非常高的效率, 几乎可以完全转化并 具有选择性. 但基团较大胺的产率较低, $\mathrm{CO}_{2}$ 和胺形成 
固体氨基甲酸盐(Scheme 5)似乎是反应效率降低的原 因 ${ }^{[46]}$. 然而, 此反应受相当复杂的空间和电子因素控 制, 而这两个因素也会影响胺的碱性强度.

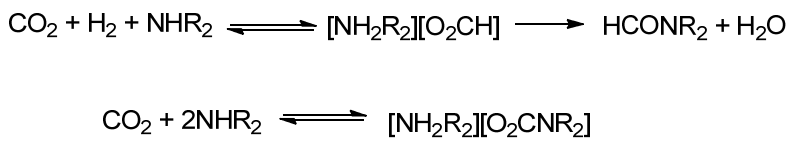

图式 5 由 $\mathrm{CO}_{2}$ 形成甲酰胺和由 $\mathrm{CO}_{2}$ 和胺形成氨基甲酸酯的 反应途径

Scheme 5 (a) Reaction pathway for the formation of formamides from $\mathrm{CO}_{2}$, and (b) carbamates from $\mathrm{CO}_{2}$ and amine

1935 年 Farlow 和 Adkins ${ }^{[47]}$ 报道了使用早期的镍催 化剂在相应的烷基胺存在下氢化 $\mathrm{CO}_{2}$ 形成甲酰胺的案 例. Han 课题组 ${ }^{[48]}$ 在 2010 年报道了使用 $\mathrm{Cu} / \mathrm{ZnO}$ 催化剂 使二甲胺在 $140{ }^{\circ} \mathrm{C} 、 1.2 \times 10^{7} \mathrm{~Pa}$ 总压力和在无溶剂条 件下 $6 \mathrm{~h}$ 以 $96 \%$ 的产率转化成 DMF. 在德国 BASF(德国 巴斯夫股份公司)的专利中, 使用了二氧化钛负载金催 化剂合成甲酰胺、DMF 和二丁基甲酰胺 ${ }^{[49,50]}$. 而非均相 催化剂的活性迄今为止还很有限.

1970 年, Haynes 等 ${ }^{[51]}$ 报道 $\mathrm{Co}, \mathrm{Rh}, \mathrm{Ir}, \mathrm{Pt}, \mathrm{Pd}, \mathrm{Ru}$ 和 $\mathrm{Cu}$ 等金属的膦络合物均相催化 $\mathrm{CO}_{2}$ 形成甲酰胺. 在 $125{ }^{\circ} \mathrm{C}$ 和 $5.5 \times 10^{6} \mathrm{~Pa}$ (室温)下反应 $17 \mathrm{~h}$ 后的 $\mathrm{TON}$ 值分 别为 $1000([\mathrm{CoH}(\mathrm{dppe})]), 1200\left(\left[\mathrm{IrCl}(\mathrm{CO})\left(\mathrm{PPh}_{3}\right)_{2}\right]\right)$ 和 900 $\left(\left[\mathrm{CuCl}\left(\mathrm{PPh}_{3}\right)_{3}\right]\right) .1994$ 年 Noyori 等 ${ }^{[52]}$ 用催化剂 $\left[\mathrm{RuCl}_{2^{-}}\right.$ $\left(\mathrm{PMe}_{3}\right)_{4}$ ]在超临界 $\mathrm{CO}_{2}$ 中将 $\mathrm{CO}_{2}$ 氢化成 DMF, 在 $100{ }^{\circ} \mathrm{C}$, $8.0 \times 10^{6} \mathrm{~Pa} \mathrm{H}_{2}, 1.3 \times 10^{7} \mathrm{~Pa} \mathrm{CO}_{2}$ 条件下反应 $19 \mathrm{~h}$ 后的最 大 $\mathrm{TON}$ 为 370000 , 基于 $\mathrm{HNMe}_{2}$ 的转化率高达 $99 \%$. Baiker 课题组 ${ }^{[53]}$ 通过使用络合物 $\left[\mathrm{RuCl}_{2}(\mathrm{dppe})_{2}\right]$ 与 $\mathrm{scCO}_{2}$ 生产 DMF 的 TOF 达 $360000 \mathrm{~h}^{-1}$. 获得 DMF 的收率高达 $85 \%$. 最近, 丁奎玲课题组 ${ }^{[54]}$ 报道了使用钌螯合物(15 17, 图 6)在 $\mathrm{CO}_{2}$ 和 $\mathrm{H}_{2}$ 中实现各种胺的 $N$-甲酰化. 催化 剂表现出非常好的效率(转化数在一个批次中高达 1940000)和在 THF 中的选择性. 通过进行简单的催化剂 再循环过程，该催化剂重复使用 12 次而没有明显损失.
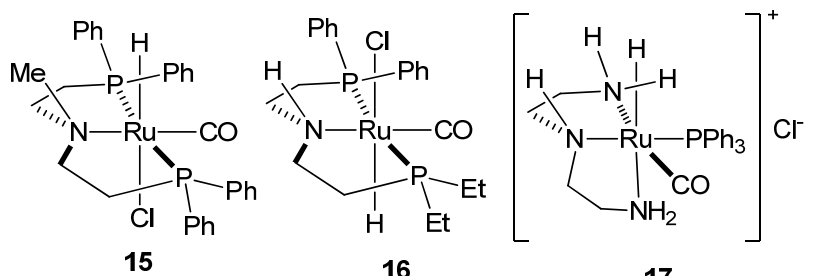

17

图 6 基于钓螯合物用于在 $\mathrm{CO}_{2}$ 和 $\mathrm{H}_{2}$ 中实现各种胺的 $N$-甲酰 化

Figure 6 Ruthenium pincer-type complexes for $\mathrm{N}$-formylation of various amines in $\mathrm{CO}_{2}$ and $\mathrm{H}_{2}$

2010 年 Beller 课题组 ${ }^{[19 b, 21,40]}$ 报道了基于铁和钴的
廉价金属催化剂, 用于催化氢化 $\mathrm{CO}_{2}$ 成 $\mathrm{DMF}$ 的研究, 该课题组用催化剂 $\mathrm{Fe}\left(\mathrm{BF}_{4}\right)_{2} \cdot 6 \mathrm{H}_{2} \mathrm{O} / \mathrm{PP}_{3} \quad\left[\mathrm{PP}_{3}=\mathrm{P}\left(\mathrm{CH}_{2}-\right.\right.$ $\left.\mathrm{CH}_{2} \mathrm{PPh}_{2}\right)_{3}$ ]以 $75 \%$ 产率催化形成甲酰胺 $(\mathrm{TON}=727)$ 和以 $41 \%$ 产率形成 $N$-甲酰基哌啶 $(\mathrm{TON}=373)$. 并用类似的 钴复合物 $\mathrm{Co}\left(\mathrm{BF}_{4}\right)_{2} \cdot 6 \mathrm{H}_{2} \mathrm{O} / \mathrm{PP}_{3}$ 在类似的反应条件下以 $73 \%$ 产率催化形成 $\mathrm{DMF}(\mathrm{TON}=1308)$ 和以 $70 \%$ 的产率 形成 $N$-甲酰基哌啶 $(\mathrm{TON}=1254)^{[21]}$. 同样, 使用含有四 齿配体三 $[$ (2-二苯基膦基)苯基 $]$ 膦的铁催化剂使 TON 高 达 4229 ${ }^{[19 b]}$. 同时也可以以 39\%产率生成二乙基甲酰胺.

\section{2 催化氢化 $\mathrm{CO}_{2}$ 成甲醛}

在 Reuss 所著的书中提到了甲醛 $\left(\mathrm{CH}_{2} \mathrm{O}\right)$ 于 1859 年 由俄罗斯化学家 Aleksandr Butlerov 首次报道 ${ }^{[55]}$. 甲醛 在商业上以环状三聚物 $1,3,5$-三惡烷或聚合形式的多聚 甲醛销售，是许多材料和有机化合物的重要前体，其水 溶液为 “福尔马林”。估计全球每年生产甲醛超过 2000 万吨并且需求仍在增长 ${ }^{[55,56]}$. 甲醛和多聚甲醛是重要的 大宗产品，广泛应用于化工、建筑、木材加工、家具制 造和纺织业 ${ }^{[55]}$. 此外, 它也应用于医药相关行业(法医/ 医院和病理实验室) 以及个人护理和消费产品. 与甲酸 类似, 甲醛也可用于储存 $\mathrm{H}_{2}$ 或 $\mathrm{CO}$.

\section{1 通过氢化催化 $\mathrm{CO}_{2}$ 合成甲醛}

2001 年, Lee 课题组 ${ }^{[57]}$ 报道了使用非均相 $\mathrm{Pt} / \mathrm{Cu} / \mathrm{SiO}_{2}$ 催化剂在 $150{ }^{\circ} \mathrm{C}$ 和 $6 \times 10^{5} \mathrm{~Pa}$ 压力下将 $\mathrm{CO}_{2}$ 氢化成甲醛的案例. 但是到目前为止, 直接由 $\mathrm{CO}_{2}$ 均相 催化氢化中制备分离甲醛仍然是一个巨大的挑战，早期 报导描述了使用 Schwartz 试剂将二氧化碳化学计量地 还原为甲醛 ${ }^{[58]}$. 反应涉及两个步骤: 形成甲醛和 $\left[\mathrm{Cp}_{2} \mathrm{Zr}(\mathrm{Cl})\right]_{2} \mathrm{O}$ 络合物 (Scheme 6), 随后分解得到 $\left[\mathrm{Cp}_{2} \mathrm{Zr}\left(\mathrm{OCH}_{3}\right)(\mathrm{Cl})\right]$ 和甲醛. 此外, Cutler 课题组 ${ }^{[59]}$ 报道了 异双金属 $\left[\mathrm{Cp}(\mathrm{CO})_{2} \mathrm{Ru}\left(\mathrm{CO}_{2}\right) \mathrm{Zr}(\mathrm{Cl}) \mathrm{Cp}_{2}\right]$ 将 $\mathrm{CO}_{2}$ 还原为甲醛 衍生物 $\left[\mathrm{Cp}(\mathrm{CO})_{2} \mathrm{Ru}\left(\mathrm{CH}_{2} \mathrm{O}\right) \mathrm{Zr}(\mathrm{Cl}) \mathrm{Cp}_{2}\right]$.

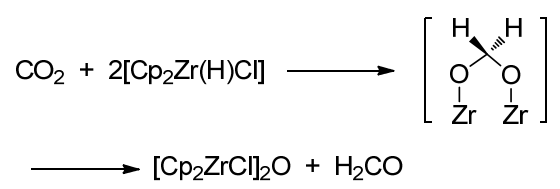

图式 6 用 Schwartz 试剂将二氧化碳按化学计量还原为甲醛 Scheme 6 Stoichiometric reduction of carbon dioxide to formaldehyde with Schwartz's reagent

2013 年, Sabo-Etienn 等 ${ }^{[60]}$ 道了在温和条件下 $(1 \times$ $10^{5} \mathrm{~Pa} \mathrm{CO}_{2}$, 室温) 以频哪醇嗍烷(B-H)作为还原剂钉催 化还原 $\mathrm{CO}_{2}$ 形成甲醛的案例. 在氢化络合物 $\left[\mathrm{RuH}_{2}\left(\mathrm{H}_{2}\right)_{2}-\right.$ $\left.\left(\mathrm{PCy}_{3}\right)_{2}\right](\mathrm{Cy}=$ 环己基 $)(\mathbf{1 8}$, Scheme 7$)$ 存在下可以观察到 几种由 $\mathrm{CO}_{2}$ 和频哪醇嗍烷形成的嗍烷化合物, 这些化合 
物被认为是形成甲酸和甲醇的中间体 ${ }^{[60]}$. 向反应溶液 中加入甲醇- $d_{4}$ 通过形成的产物 $\mathrm{CD}_{3} \mathrm{OCH}_{2} \mathrm{OD}$ 可以证明 甲醛被 “捕获” (Scheme 7).

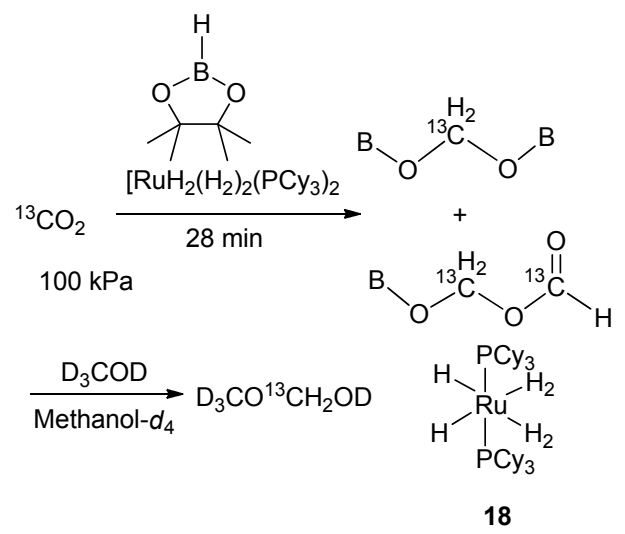

图式 7 钉催化的 $\mathrm{CO}_{2}$ 还原为甲醛衍生物

Scheme 7 Ruthenium-catalyzed reduction of $\mathrm{CO}_{2}$ to formaldehyde derivatives

一年后, 此课题组 ${ }^{[60]}$ 报道了钉络合物 $\left[\mathrm{RuH}_{2}\left(\mathrm{H}_{2}\right)_{2}\right.$ $\left.\left(\mathrm{PCyp}_{3}\right)_{2}\right](\mathrm{Cyp}=$ 环戊基 $)$ 催化 $\mathrm{CO}_{2}$ 还原为甲醛并进行分 离的案例. Sabo-Etienne 和同事在温和条件 $\left(1 \times 10^{5} \mathrm{~Pa}\right.$ $\mathrm{CO}_{2}$, 室温)先将其还原为亚胺, 随后亚胺在水中水解为 相应的胺和甲醛(Scheme 8).

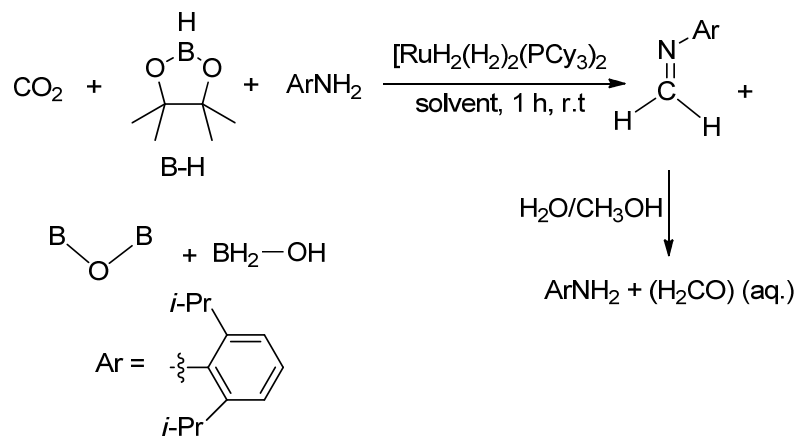

图式 8 钉催化 $\mathrm{CO}_{2}$ 还原成原位捕获的甲醛并发生水解

Scheme 8 Ruthenium-catalyzed reduction of $\mathrm{CO}_{2}$ to in situ trapped formaldehyde and subsequent hydrolysis

2014 年, Prechtl 及同事 ${ }^{[61]}$ 报道了使用甲醛水溶液和 多聚甲醛溶液作为储氢介质的案例. 该课题组使用在空 气中稳定的钉催化剂 $\left[(\mathrm{Ru}(p \text {-cymene }))_{2}(m-\mathrm{Cl})_{2} \mathrm{Cl}_{2}\right]$, 使得 甲醛和多聚甲醛的分解可以在无碱条件下进行. 该催化 方法耐受氧并可得到纯的 $\mathrm{CO}_{2}$ 和 $\mathrm{H}_{2}$ 混合物. 在 $95{ }^{\circ} \mathrm{C}$ 时 反应 $60 \mathrm{~min}$ 以 $84 \%$ 的产率得到 $\mathrm{H}_{2}$. 甲醛和多聚甲醛在 水中反应得到水合醛 $\mathrm{H}_{2} \mathrm{C}(\mathrm{OH})_{2}$, 由此相比甲酸(氢的质 量分数 $4.4 \%$ ) 得到较高氢含量的分子(质量分数 $8.4 \%$ ). 由于在形成水合醛中间体涉及了水, 这种方法可以被认 为是化学上间接的水分解系统, 其中水用作质子源和作 为氢化物源最后形成 $\mathrm{H}_{2}$ (Scheme 9).

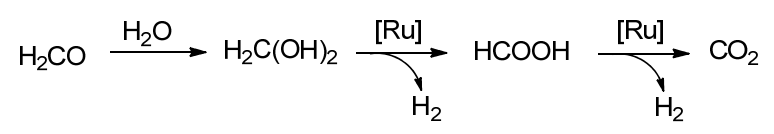

图式 9 在甲醛和水中进行钉催化氢化

Scheme 9 Ruthenium-catalyzed hydrogen formation from formaldehyde and water

\section{3 催化氢化 $\mathrm{CO}_{2}$ 成甲醇}

甲醇在室温下是液体 $\left(\mathrm{m} . \mathrm{p} .=-97.6{ }^{\circ} \mathrm{C}\right)$, 是化学工 业最重要的商品之一，2013 年有 6500 万吨甲醇被消

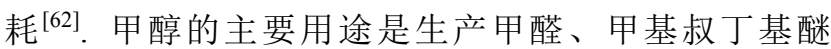
(MTBE) 和叔戊基甲基醚以及乙酸. 甲醇还可以生产二 甲醚、烯烃、甲胺、甲基丙烯酸甲酯和氯甲烷 ${ }^{[62,64,65]} .1986$ 年 Asinger ${ }^{[65]}$ 提出了偶合能量和物质价值链以甲醇作为 中心分子的观点. 2005 年, Olah 等 ${ }^{[66]}$ 提出了一种基于类 似的 “甲醇经济” 的观点. 他最近的一本书提供了关于 当前技术和可能的未来前景的详细概述.

\section{1 通过催化氢化 $\mathrm{CO}_{2}$ 和 $\mathrm{H}_{2}$ 合成甲醇}

\subsection{1 非均相催化氢化 $\mathrm{CO}_{2}$ 和 $\mathrm{H}_{2}$ 合成甲醇}

当将 $\mathrm{CO}_{2}$ 加入到合成气流中以调节 $\mathrm{C} / \mathrm{H}$ 平衡时，通 过非均相催化剂将 $\mathrm{CO}_{2}$ 和 $\mathrm{H}_{2}$ 转化为甲醇已经作为当前 甲醇生产工艺的重要部分. 估计每年约有 200 万吨二氧 化碳以这种方式转化为甲醇 ${ }^{[67]}$. 目前已知的可以通过 催化剂催化氢化的反应有 $\mathrm{CO}$ 和 $\mathrm{CO}_{2}$ 转化为甲醇以及水 煤气变换反应(WGS: $\left.\mathrm{CO}+\mathrm{H}_{2} \mathrm{O} \rightarrow \mathrm{CO}_{2}+\mathrm{H}_{2}\right)^{[62]}$, 特别是 基于 $\mathrm{Cu} / \mathrm{Zn}$ 氧化物的催化剂. 并且向反应中加入少量 $(3 \%)$ 的 $\mathrm{CO}_{2}$ 气体可以提高甲醇的产率 ${ }^{[68]}$. 但是, 大量的 $\mathrm{CO}_{2}$ 或纯 $\mathrm{CO}_{2}$ 会导致水的形成并降低甲醇的形成速 率 ${ }^{[69,70]}$.

1994 年, 在 Sued-Chemie(现为 Clariant)的试点工厂 已经开始使用 $\mathrm{Cu} / \mathrm{Zn} / \mathrm{Al}$ 催化剂在 $6.0 \times 10^{6} \mathrm{~Pa}$ 压力和 $260{ }^{\circ} \mathrm{C}$ 条件下将 $\mathrm{CO}_{2}$ 氢化成甲醇 ${ }^{[71]}$. 得到甲醇的转化率 约为 35\% 45\%, 选择性高达 99.96\%(不含水). 2012 年, Behrens 等 ${ }^{[72]}$ 报道了用多成分催化剂材料 $\mathrm{Cu} / \mathrm{ZnO} / \mathrm{Al}_{2} \mathrm{O}_{3}$ 将 $\mathrm{CO}$ 和 $\mathrm{CO}_{2}$ 转化为甲醇，在 $6.0 \times 10^{6} \mathrm{~Pa}$ 合成气混合物 (59.5\% $\mathrm{H}_{2}, 8 \% \mathrm{CO}_{2}, 6 \% \mathrm{CO}$, 加惰性气体)和 $210 \sim$ $250{ }^{\circ} \mathrm{C}$ 条件下 $\mathrm{TOF}$ 为 $75.6 \mathrm{~h}^{-1}$. 同位素标记实验表明由 $\mathrm{CO} / \mathrm{CO}_{2} / \mathrm{H}_{2}$ 合成气混合物形成的甲醇主要来自 $\mathrm{CO}_{2}{ }^{[73]}$.

\subsection{2 均相催化氢化 $\mathrm{CO}_{2}$ 和 $\mathrm{H}_{2}$ 合成甲醇}

与非均相催化氢化 $\mathrm{CO}_{2}$ 和 $\mathrm{H}_{2}$ 合成甲醇相比, 均相 催化氢化 $\mathrm{CO}_{2}$ 和 $\mathrm{H}_{2}$ 合成甲醇过去研究报道的不多, 但 从 1993 年 Sasaki 等 ${ }^{[74]}$ 第一次报道了金属有机 $\mathrm{Ru}_{3}(\mathrm{CO})_{12}-\mathrm{KI}$ 催化剂体系催化 $\mathrm{CO}_{2}$ 和 $\mathrm{H}_{2}$ 形成甲醇以来, 已经成为目前研究的热点. 2011 年, Milsteins 课题 组 ${ }^{[75 ~ 77]}$ 报道了通过氢化 $\mathrm{CO}_{2}$ 衍生的稳定中间体实现了 
由 $\mathrm{CO}_{2}$ 间接转化为甲醇的多步路线(Scheme 10). 他们证 明了多功能的 Ru-PNN 钳形络合物 19 可以使甲酸甲酯、

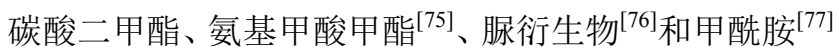
有效氢化而得到产物甲醇. 由于这些底物可以由 $\mathrm{CO}_{2}$ 生 产，因此可以设想通过有机金属催化 $\mathrm{CO}_{2}$ 生产甲醇的间 接合成路线. 其中, 通过使用 Ru-PNN 钳形络合物可以 将碳酸二甲酯定量地氢化为甲醇, 在 $6.0 \times 10^{6} \mathrm{~Pa} \mathrm{H}_{2}$, $145{ }^{\circ} \mathrm{C}$ 条件下 $\mathrm{TOF}$ 高达 $2500 \mathrm{~h}^{-1}$. 并可用相同的络合物 在 $5.0 \times 10^{6} \mathrm{~Pa} \mathrm{H}_{2}, 110{ }^{\circ} \mathrm{C}$ 条件下氢化甲酸甲酯获得高达 $531 \mathrm{~h}^{-1}$ 的 $\mathrm{TOF}^{[77]}$.
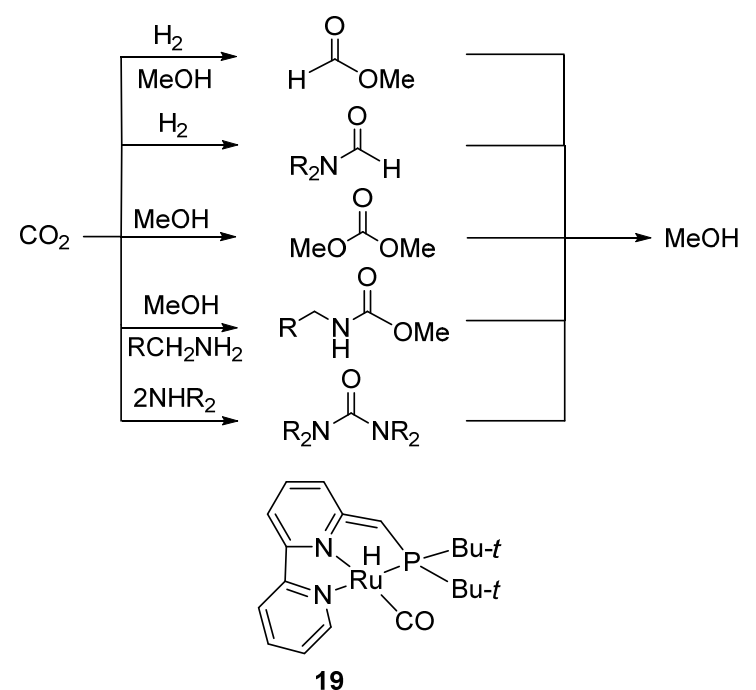

图式 10 从 $\mathrm{CO}_{2}$ 合成甲醇的间接途径

Scheme 10 Indirect routes for methanol synthesis from $\mathrm{CO}_{2}$

丁奎岭课题组 ${ }^{[78]}$ 提出了一种通过碳酸亚乙酯间接生产 甲醇的方法(Scheme 11), 从而导致了壳牌 Omega 生产 工艺的革新. 在 Omega 方法中, 通过在第一步先由环氧 乙烷和 $\mathrm{CO}_{2}$ 产生的碳酸亚乙酯, 之后再水解产生乙二 醇. 而丁奎岭课题组提出通过氢化来替代水解步骤可以 同时生产甲醇. 通过使用 Ru-PNP 型催化剂 $\mathbf{2 0}$ 使得生成 甲醇的 TON 高达 87000, TOF 高达 $1200 \mathrm{~h}^{-1}$.

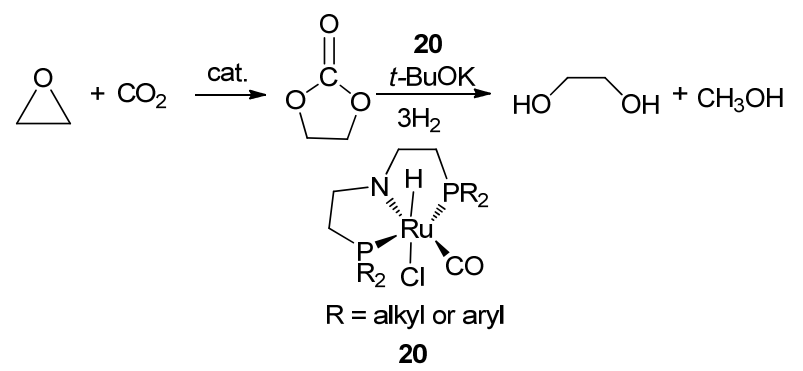

图式 11 有机金属化合物催化氢化 $\mathrm{CO}_{2}$ 形成乙二醇和甲醇 Scheme 11 Catalytic hydrogenation of $\mathrm{CO}_{2}$ to ethylene glycol and methanol by organometallic compounds

2011 年, Sanford 课题组 ${ }^{[43]}$ 将 Milstein 的甲酸酯氢化

催化剂结合到一锅级联反应中，他们将 Milstein 催化剂 与另一种 $\mathrm{Ru}$ 络合物和路易斯酸(Scheme 12, 21 和 22) 一 起组成三组分催化剂体系, 但效率没有想象的高. 2015 年, 此课题组 ${ }^{[79]}$ 报道了类似的将 $\mathrm{CO}_{2}$ 氢化成甲醇的的级 联反应方法, 其中 DMF 作为中间体而形成(Scheme 12, 21). 在 $\mathrm{HNMe}_{2}$ 存在下由 $\mathrm{CO}_{2}$ 氢化成 $\mathrm{DMF}$ 具有较高的 活性和选择性. 此反应的后一步是甲酰胺氢化成甲醇, 而此反应的文献报道较少. Sanford 和同事也报道了在 $5.0 \times 10^{6} \mathrm{~Pa} \mathrm{H}_{2}$ 压力和 $155{ }^{\circ} \mathrm{C}$ 下使用 $\mathrm{Ru}-\mathrm{MACHO}-\mathrm{BH}_{4}$ 络合物和碱 $\mathrm{K}_{3} \mathrm{PO}_{4}$ (50 equiv.)选择性氢化 DMF 至甲醇的 案例.

$$
\mathrm{CO}_{2} \stackrel{\mathrm{H}_{2}}{\longrightarrow} \mathrm{HCO}_{2} \mathrm{H} \underset{-\mathrm{H}_{2} \mathrm{O}}{\longrightarrow} \mathrm{HCO}_{2} \mathrm{R} \underset{-\mathrm{ROH}}{\stackrel{2 \mathrm{H}_{2}}{\longrightarrow}} \mathrm{CH}_{3} \mathrm{OH}
$$

(a)

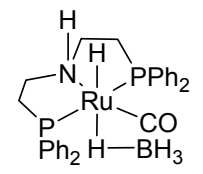

21 (b)

$\mathrm{Sc}(\mathrm{OTf})_{3}$

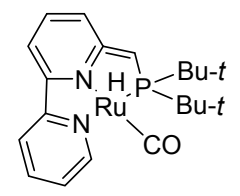

22

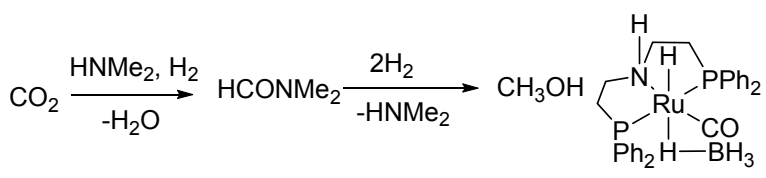

21

图式 12 通过甲酸烷基酯(上)和 $\mathrm{DMF}$ (下)将 $\mathrm{CO}_{2}$ 氢化成甲醇 的级联反应

Scheme 12 Cascade reactions for the hydrogenation of $\mathrm{CO}_{2}$ to methanol via alkyl formate (upper) and DMF (lower)

最近, Olah 和 Prakash 课题组 ${ }^{[80]}$ 将均相 $\mathrm{Ru}$ 催化剂 (图 7, 21，23 26) 用于通过使用多胺碱来形成氨基甲酸 加合物作为反应中的实际底物, 从而由 $\mathrm{CO}_{2}$ 和 $\mathrm{H}_{2}$ 合成 甲醇. 多胺可用从只含有约 $400 \mathrm{mg} / \mathrm{L}$ 的 $\mathrm{CO}_{2}$ 气体混合 物中捕获 $\mathrm{CO}_{2}$. 这使得使用均相催化剂从空气中捕获 $\mathrm{CO}_{2}$ 并直接催化成甲醇成为可能. 其报道从空气中捕获 的 $\mathrm{CO}_{2}$ 形成 $\mathrm{CH}_{3} \mathrm{OH}$ 的产率高达 $79 \%{ }^{[80]}$.

2012 年, Klankermayer, Leitner 等 ${ }^{[81]}$ 报道了整合甲 酸烷基酯的级联反应(Scheme 13); 催化剂体系是钉金 属中心和三齿配体 triphos [triphos $=1,1,1$-三（二苯基膦 基甲基)乙烷]. 催化剂 $\left[\mathrm{Ru}(\right.$ triphos)(tmm) $] / \mathrm{HNTf}_{2}\left[\mathrm{HNTf}_{2}\right.$ $=$ 双 (三氟甲烷)磺酰胺, tmm =三甲基甲烷](28, Scheme 13)使得 $\mathrm{CO}_{2}$ 在 $\mathrm{THF} / \mathrm{EtOH}$ 溶剂中氢化为甲醇的 $\mathrm{TON}$ 高 达 $221\left(2.0 \times 10^{6} \mathrm{~Pa} \mathrm{CO}_{2}, 6.0 \times 10^{6} \mathrm{~Pa} \mathrm{H}_{2}, 140{ }^{\circ} \mathrm{C}\right)$. 同位 素标记实验证明甲醇是由 $\mathrm{CO}_{2}$ 和 $\mathrm{H}_{2}$ 催化产生的, 并且使 
<smiles>O=C(c1ccccc1)P1(c2ccccc2)(c2ccccc2)C2CCN(CC2)P1c1ccccc1</smiles>

21<smiles>CCS[R]1(Cl)NCCS1</smiles>

24

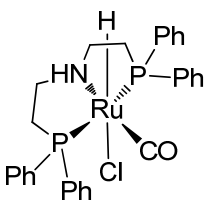

22

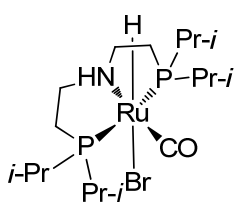

23

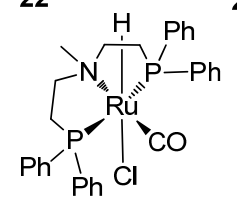

25
图 7 几种均相的 $\mathrm{Ru}$ 催化剂催化氢化 $\mathrm{CO}_{2}$ 形成 $\mathrm{CH}_{3} \mathrm{OH}$

Figure 7 Several homogeneous $\mathrm{Ru}$ catalysts catalyze the hydrogenation of $\mathrm{CO}_{2}$ to form $\mathrm{CH}_{3} \mathrm{OH}$

用该催化剂体系也可以进行甲醇的逆向反应. 该催化体 系是基于已发现的催化剂体系 $\left[\mathrm{Ru}(\mathrm{acac})_{3}\right]$ 和 triphos 或 $\left[\mathrm{Ru}(\right.$ triphos)(tmm) $]$ 在酸性助催化剂存在下 ${ }^{[82]}$ 将羧酸酯氢

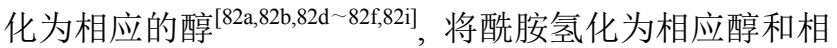
应的胺 ${ }^{[82 c, 82 g, 82 i]}$, 将碳酸酯转化为相应的醇以及将㽷衍生 物转化为相应的胺和甲醇 ${ }^{[82 i]}$. 因此也可以使用催化剂体 系 27 或 28 将甲酸甲酯和甲酸乙酯氢化成相应的醇, 相关 该体系的研究已有综述 ${ }^{[83]}$.

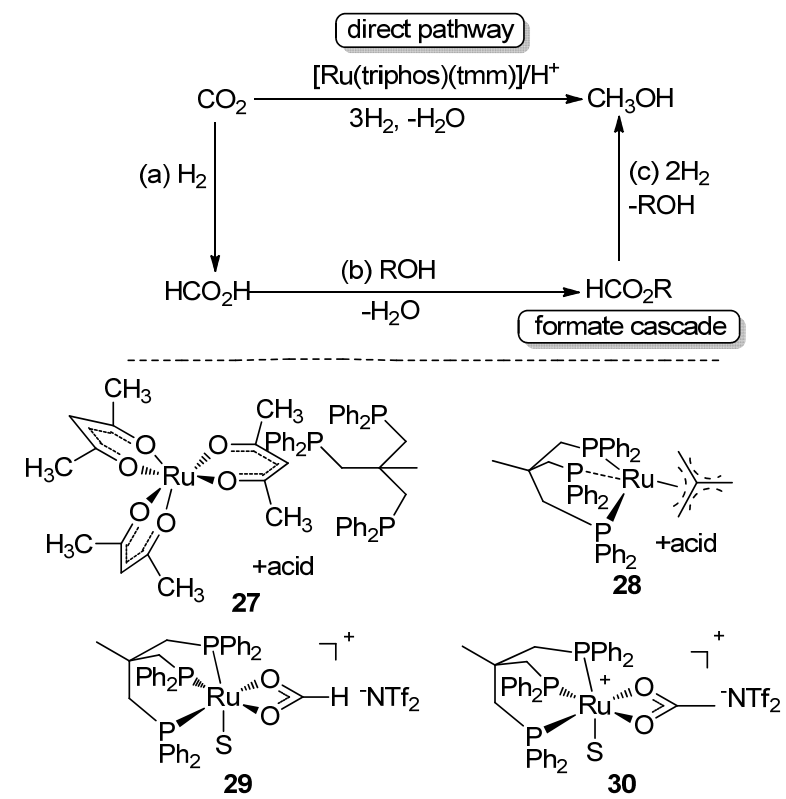

图式 13 通过甲酸酯将 $\mathrm{CO}_{2}$ 氢化为甲醇

Scheme 13 Hydrogenation of $\mathrm{CO}_{2}$ to methanol through a formate ester cascade

详细的机理研究表明, $\mathrm{CO}_{2}$ 到甲醇的多步转化在没 有甲酸烷基酯中间体形成时也可以直接在 Ru-triphos 中 心上发生, 从而报道了直接从 $\mathrm{CO}_{2} / \mathrm{H}_{2}$ 选择性形成甲醇 的催化过程 ${ }^{[84]}$. 在无醇添加剂的情况下使用 $[\mathrm{Ru}$ (triphos)(tmm)] $/ \mathrm{HNTf}_{2}$ (28)反应 $64 \mathrm{~h}$ 后甲醇 TON 高达 895 $\left(\mathrm{CO}_{2} / \mathrm{H}_{2}=1: 3,1.2 \times 10^{7} \mathrm{~Pa}\right.$ 总压, $\left.140{ }^{\circ} \mathrm{C}\right)$. 高压原位
NMR 揭示了阳离子甲酸盐络合物 $\left[\mathrm{Ru}\left(\eta^{2}-\mathrm{O}_{2} \mathrm{CH}\right)-\right.$ (triphos) $(\mathrm{S})] \mathrm{NTf}_{2}(29, \mathrm{~S}=$ 自由配位点或溶剂) 在溶液中 成为催化活性中间体. 基于这一发现, 开发了类似的乙 酸基络合物 $\left[\mathrm{Ru}\left(\eta^{2}-\mathrm{OAc}\right)(\right.$ triphos) $(\mathrm{S})] / \mathrm{NTf}_{2}$ (30)作为前体. 在无任何酸和醇添加剂情况下, 用催化剂前体 $\mathbf{3 0}$ 进行 $\mathrm{CO}_{2}$ 氢化得到的 $\mathrm{TON}$ 为 $165\left(2.0 \times 10^{6} \mathrm{~Pa} \mathrm{CO}_{2}, 6.0 \times 10^{6}\right.$ $\left.\mathrm{Pa} \mathrm{H}_{2}, 140{ }^{\circ} \mathrm{C}\right)$, 因此表明酸添加剂仅仅是产生阳离子 活性络合物, 正如 NMR 观察到的 $\left[\mathrm{Ru}\left(\eta^{2}-\mathrm{O}_{2} \mathrm{CH}\right)-\right.$ (triphos)(S) $]^{+}$.

2017 年, Beller 课题组 ${ }^{[86]}$ 报道了第一个使用非贵金 属催化剂将 $\mathrm{CO}_{2}$ 均相氢化为甲醇的案例. 他们使用钴催 化剂 $\left[\mathrm{Co}(\mathrm{acac})_{3}\right]$ 、配体 Triphos 和路易斯酸 $\mathrm{HNTf}_{2}$, 在 $7.0 \times 10^{6} \mathrm{~Pa} \mathrm{H}_{2}, 2.0 \times 10^{6} \mathrm{~Pa} \mathrm{CO}_{2}$ 和 $100{ }^{\circ} \mathrm{C}$ 条件下 $\mathrm{THF} /$ $\mathrm{EtOH}$ 溶剂中反应 $96 \mathrm{~h}$ 后生成甲醇的 TON 为 78 .

\section{4 催化氢化 $\mathrm{CO}_{2}$ 作为 $\mathrm{C}_{1}$ 合成子的反应}

2000 年, Tominaga 和 Sasaki ${ }^{[87]}$ 报道了用 $\mathrm{CO}_{2}$ 和 $\mathrm{H}_{2}$ 进行烯烃的 $\mathrm{C}_{1}$ 延长反应(Scheme $14, \mathrm{a}$ ), 在 $\mathrm{LiCl}$ 的存在
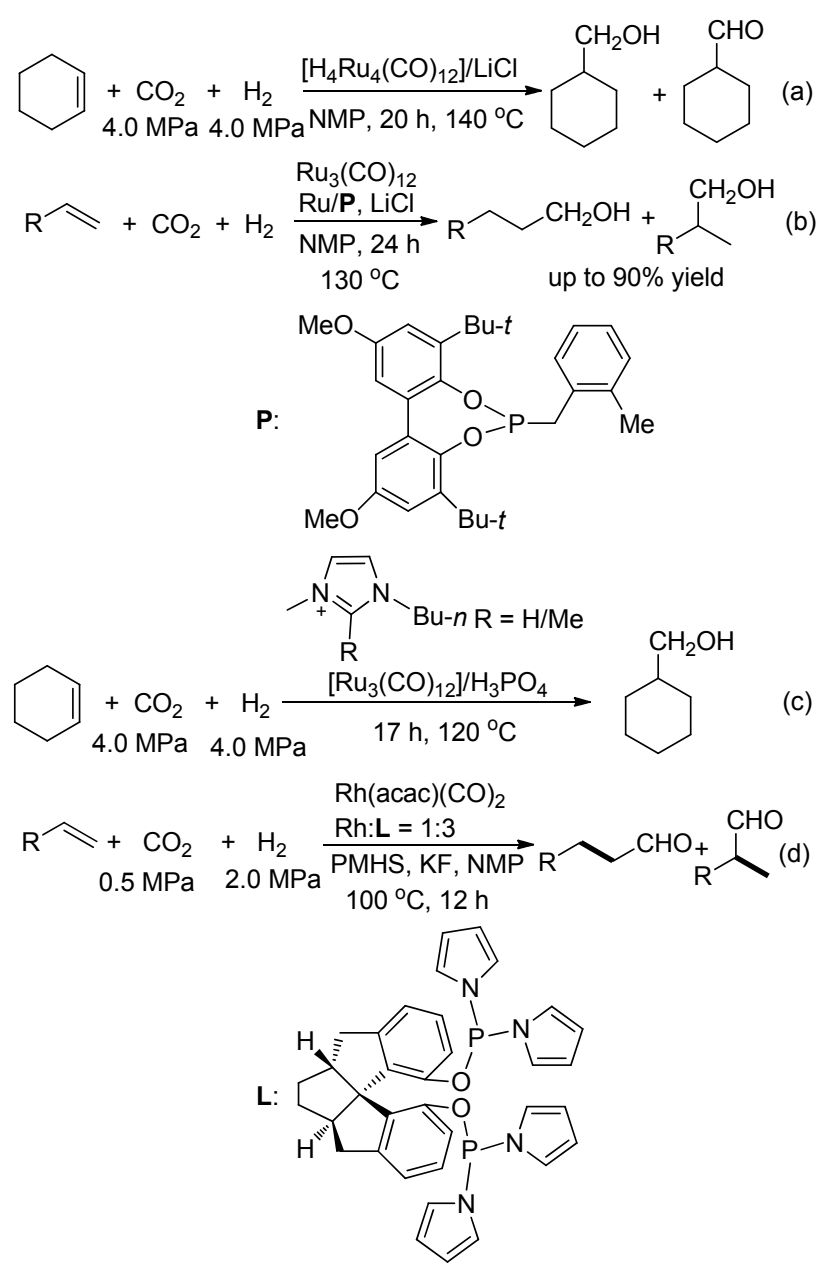

图式 14 钓催化烯烃与 $\mathrm{CO}_{2} / \mathrm{H}_{2}$ 的羟甲基化/加氢甲酰化反应 Scheme 14 Ruthenium/Rhodium catalytic hydroxymethylated/hydroformylated the olefins with $\mathrm{CO}_{2} / \mathrm{H}_{2}$ 
下使用钓催化剂 $\left[\mathrm{H}_{4} \mathrm{Ru}_{4}(\mathrm{CO})_{12}\right.$ 可以由环已烯、 $\mathrm{CO}_{2}$ 和 $\mathrm{H}_{2}$ 以 $88 \%$ 的产率获得羟甲基环己烷. 2014 年 Beller 课题 组 ${ }^{[88}$ 报道了使用高位阻的亚磷酸酯配体作为改进催化 体系效率的方法 (Scheme 14, b), 通过使用钓催化剂 $\left[\mathrm{Ru}_{3}(\mathrm{CO})_{12}\right]$ 在较低温度下将 $\mathrm{CO}_{2} / \mathrm{H}_{2}$ 更有效地和内烯烃 进行羟甲基化. 相比无配体的催化剂体系, 所需醇的产 率得到明显改善. 同年, Dupont 等 ${ }^{[89]}$ 也报道了使用催化 剂 $\left[\mathrm{Ru}_{3}(\mathrm{CO})_{12}\right.$ 在催化量的卤素基离子液体 $[\mathrm{BMIM}][\mathrm{Cl}]$ (BMIM: 1-丁基-3-甲基咪唑鍮)存在下取得了很好的活 性(Scheme 14, c). 2017 年, 丁奎岭课题组 ${ }^{[85]}$ 报道了使用 铑催化剂 $\mathrm{Rh}(\mathrm{acac})(\mathrm{CO})_{2}$ 和配体 $\mathbf{L}$ (Scheme 14)在聚甲基 氢硅氧烷(PMHS)、 $\mathrm{KF}$ 和路易斯碱 $N$-甲基吡咯烷酮 (NMP) 存在下和 $2.0 \times 10^{6} \mathrm{~Pa} \mathrm{H}_{2}$ 和 $5 \times 10^{5} \mathrm{~Pa} \mathrm{CO}_{2}$ 条件下 催化 $\mathrm{CO}_{2}$ 和烯烃, $100{ }^{\circ} \mathrm{C}$ 下反应 $12 \mathrm{~h}$ 的 TON 最高达 1000000 (Scheme 14, d).

2009 年, Srivastava 和 Eilbracht ${ }^{[90]}$ 报道了使用催化剂 体系 $\left[\mathrm{Ru}_{3}(\mathrm{CO})_{12}\right] / \mathrm{LiCl} / \mathrm{BTAC}(\mathrm{BTAC}=$ 苄基三甲基氯化 铵)(催化 $\mathrm{CO}_{2} / \mathrm{H}_{2}$ 和一系列烯烃以 $35 \% \sim 98 \%$ 的产率生成 仲胺和叔胺的氨基甲基化反应(Eq. 1). 2013 年, Klankermayer 和同事 ${ }^{[91]}$ 报道了使用钉催化剂 [ $\{\mathrm{RuCl}-$ $\left.\left.(\mathrm{CO})_{2}\right\}_{2}\right] / \mathrm{PPh}_{3}$ 将未活化的烯烃、 $\mathrm{CO}_{2}$ 和 $\mathrm{H}_{2}$ 直接 催化形成着酸的案例(Scheme 15, a), 此催化剂体系具有
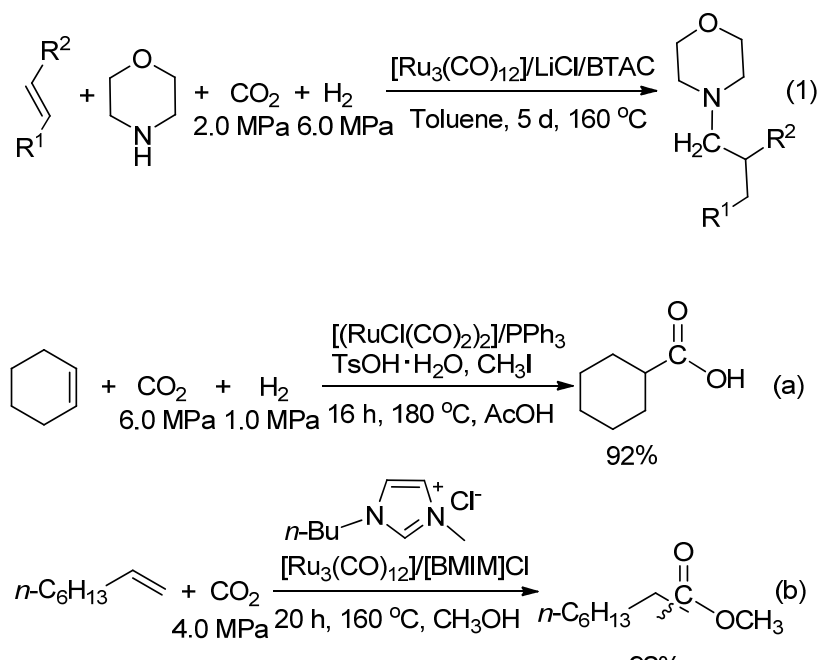

$92 \%$

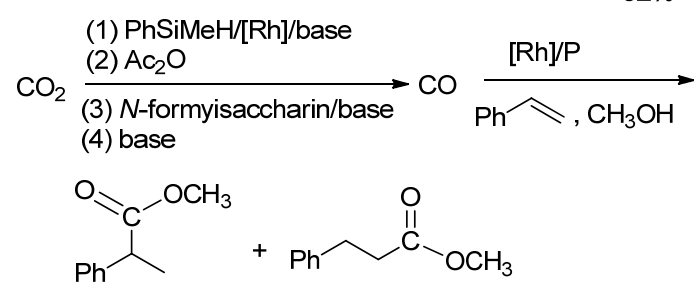

(c)

图式 15 钉/错催化烯烃与 $\mathrm{CO}_{2} / \mathrm{H}_{2}$ 的羟基羰基化/烷氧基羰基 化反应

Scheme 15 Ruthenium/rhodium catalyzes the hydrolysis of olefins and $\mathrm{CO}_{2} / \mathrm{H}_{2}$ hydroxycarbonylated/alkoxycarbonylated reactions
较高的转化率和选择性. 2014 年, Beller 课题组 ${ }^{[92]}$ 报道了 使用催化剂体系 $\left[\mathrm{Ru}_{3}(\mathrm{CO})_{12}\right] /[\mathrm{BMIM}] \mathrm{Cl}$ 将烯烃、 $\mathrm{CO}_{2}$ 和 醇直接转化成羧酸酯的反应(Scheme 15, b). 2015 年 Fleischer 等 ${ }^{[93}$ 报道了间接利用 $\mathrm{CO}_{2}$ 作为 $\mathrm{C}_{1}$ 源转化为 $\mathrm{CO}$ 后应用于不同烯烃的烷氧基羰基化反应(Scheme 15, c), 生成相应的酯产物的产率可达 $92 \%$.

2013 年, Klankermayer 和同事 ${ }^{[94]}$ 报道了以 $\mathrm{CO}_{2} / \mathrm{H}_{2}$ 作为结构单元对伯胺和仲胺直接 $N$-甲基化的第一个例 子. 通过使用催化剂体系 $\left[\mathrm{Ru}(\right.$ triphos)(tmm) $] / \mathrm{HNTf}_{2}$ 实现 $\mathrm{CO}_{2} / \mathrm{H}_{2}$ 对芳香族胺的直接 $\mathrm{N}$-甲基化, 显示出较高的反 应活性(Scheme 16, a). 同年 Beller 课题组 ${ }^{\left[{ }^{[5]}\right.}$ 也报道了使 用催化剂体系 $\left[\mathrm{Ru}(\mathrm{acac})_{3}\right] /$ triphos 在 $\mathrm{LiCl}$ 存在下实现 $\mathrm{CO}_{2} / \mathrm{H}_{2}$ 对芳香族和脂肪族胺的直接 $N$-甲基化的案例 (Scheme 16, b).

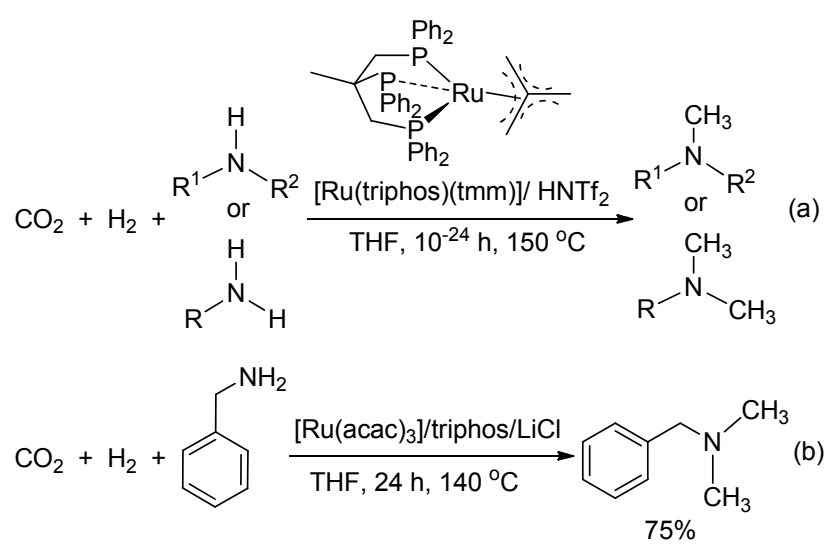

图式 16 钉催化 $\mathrm{CO}_{2} / \mathrm{H}_{2}$ 对芳香族和脂肪族胺的 $N$-甲基化 Scheme $16 N$-Methylation of aromatic and aliphatic amines by $\mathrm{CO}_{2} / \mathrm{H}_{2}$ catalyzed by ruthenium

2013 年 Klankermayer 等 ${ }^{[96]}$ 报道了催化剂体系 $[\mathrm{Ru}$ (triphos)(tmm) $] / \mathrm{HNTf}_{2}$ 能够在相应的反应条件下对 甲酰胺进行氢化和 $N$-甲基化，作为获得不对称甲基/烷 基叔胺的途径(Scheme 17, a), 在标准反应条件和 $2.0 \times$ $10^{6} \mathrm{~Pa} \mathrm{CO}_{2}, 6.0 \times 10^{6} \mathrm{~Pa} \mathrm{H}_{2}$ 条件下转化为 $N$-乙 基- $N$-甲 基苯胺的产率为 $69 \%$. 2014 年 Leitner 课题组 ${ }^{[97]}$ 报道了使 用相同的催化剂体系 $\left[\mathrm{Ru}(\right.$ triphos)(tmm) $] / \mathrm{HNTf}_{2}$ 实现了 对 $N$-(1-苯基亚乙基)苯胺的 $N$-甲基化, 该催化剂体系可 以获得最高 $96 \%$ 的产率(Scheme 17, b). 催化剂体系 $[\mathrm{Ru}$ (triphos)(tmm) $] / \mathrm{HNTf}_{2}$ 也可用于伯胺、羰基底物和 $\mathrm{CO}_{2} / \mathrm{H}_{2}$ 的氢化和 $N$-甲基化反应. 在标准反应条件下苯 胺衍生物和芳族酫可以以较高的产率获得胺. 而芐胺则 需要提高反应温度 $\left(160{ }^{\circ} \mathrm{C}\right)$ 来得到产物(Scheme 17, c).

上述钓催化的氢化 $/ N$-甲基化方法被成功地应用于 抗真菌剂布替芸芬 ( $N$-(4-叔丁基茮基)- $N$-甲基-1-萗胺)的 合成(Eq. 2) ${ }^{[97]}$. 通过 $\mathrm{CO}_{2} / \mathrm{H}_{2}$ 对价廉底物 1-菜甲醛和 4叔丁基芐胺的甲基化得到布替萗芬产物. 从分离的亚胺 可以以 $88 \%$ 的产率得到产物，也可以三组份偶联以 $60 \%$ 


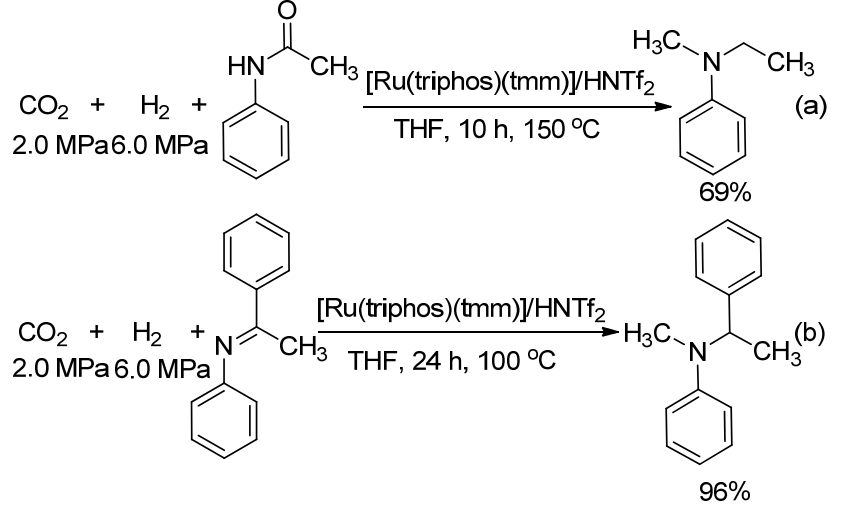

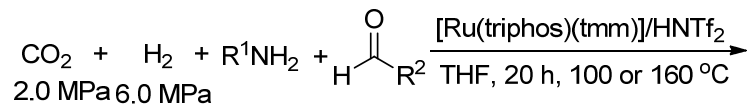<smiles>[R]CN([R])C</smiles>

(c)

图式 17 钉催化 $\mathrm{CO}_{2} / \mathrm{H}_{2}$ 对乙酰苯胺的氢化 $/ N$-甲基化、钉催 化 $\mathrm{CO}_{2} / \mathrm{H}_{2}$ 对亚胺的甲基化及钉催化伯胺、羰基底物和 $\mathrm{CO}_{2} / \mathrm{H}_{2}$ 的氢化 $/ N$-甲基化反应

Scheme 17 Ruthenium-catalyzed $\mathrm{CO}_{2} / \mathrm{H}_{2}$ hydrogenation of acetanilide/ $N$-methylation, ruthenium-catalyzed $\mathrm{CO}_{2} / \mathrm{H}_{2}$ methylation of imines and ruthenium-catalyzed the hydrogenation $/ \mathrm{N}$-methylation of primary amines, carbonyl substrates and $\mathrm{CO}_{2} / \mathrm{H}_{2}$

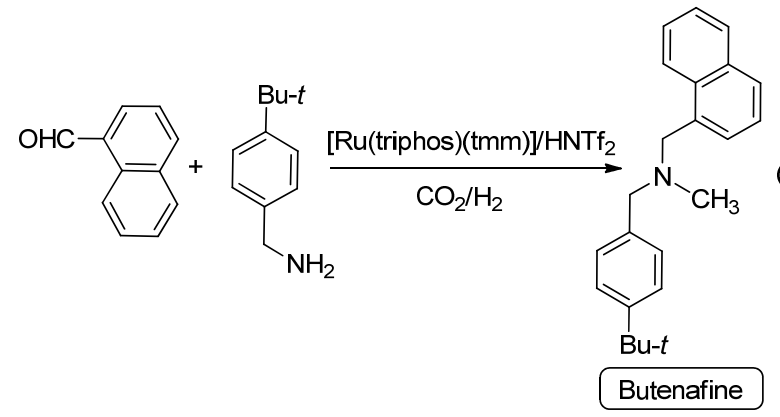

的产率得到产物. 相比由对叔丁基苯甲酸作为底物进行 四步反应的常规方法, 该方法减少了反应步骤, 提高了 原子经济百分数(达 $85 \%$ ), 并很好地说明了 $\mathrm{CO}_{2} / \mathrm{H}_{2}$ 作为 $\mathrm{C}_{1}$ 合成子的合成潜力, 符合 “绿色化学” 的指标.

除了从 $\mathrm{N}-\mathrm{H}$ 键上引入甲基以外, 2014 年 Beller 课 题组 ${ }^{[98]}$ 报道了第一个由 $\mathrm{CO}_{2} / \mathrm{H}_{2}$ 进行 $\mathrm{C}-\mathrm{H}$ 键甲基化的 实例(Eq. 3). 通过使用催化剂体系 $\left[\mathrm{Ru}(\mathrm{acac})_{3}\right] /$ triphos 并

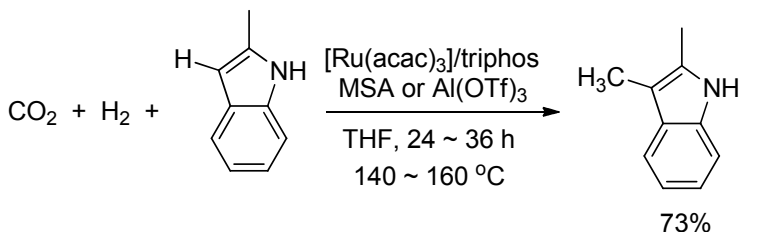

以甲磺酸 $(\mathrm{MSA})$ 和 $\mathrm{Al}(\mathrm{OTf})_{3}$ 作为路易斯酸用于富电子杂 芳族化合物的 $\mathrm{C}-\mathrm{H}$ 键甲基化, 并表现出较高的反应产 率.

\section{5 总结与展望}

综述了近年来金属有机络合物催化的氢化 $\mathrm{CO}_{2}$ 合 成甲酸、盐及衍生物、甲醛、甲醇及作为 $\mathrm{C}_{1}$ 合成子合 成一些高附加值精细化学品的研究进展. 甲酸、盐及衍 生物、甲醛、甲醇这些可以基于 $\mathrm{CO}_{2}$ 生产的基础化学品, 除用作化学工业的原料外, 还具有作为能量载体或储存 的潜力. 因此作为 $\mathrm{CO}_{2}$ 减排和资源利用的潜力巨大. 尽 管目前由于成本问题，甲酸、盐及衍生物、甲醛、甲醇 这些基础化学品还没有完全由 $\mathrm{CO}_{2}$ 氢化来合成, 但处于 降低 $\mathrm{CO}_{2}$ 温室气体排放的考虑, 研究来自可持续能源氢 气的输入，作为未来能源使用，对于充分发挥这种途径 的生态效益是非常必要的. 更为重要的是, 通过均相催 化氢化 $\mathrm{CO}_{2}$ 在某一特定区域引入产生 $\mathrm{C}_{1}$ 结构单元, 开 辟了得到重要的官能团化合物如羧酸、醛、醇和胺等高 附加值精细化学品新的绿色合成途径. 这些反应建立了 能够用于合成具有生物活性复杂分子的高选择性和清 洁的合成方法. 引入甲基就是一个很好的例子，使用 $\mathrm{CO}_{2} / \mathrm{H}_{2}$ 不仅代替了对环境不太友好的试剂, 而且提供 了一种新的绿色合成方法. 由于 $\mathrm{CO}_{2}$ 和 $\mathrm{H}_{2}$ 是相对低成 本的原料, 它们的靶向使用不仅可以为生产更高附加值 化学品的可持续生产提供直接的经济驱动力. 而且可以 替代环境不太友好的试剂, 使得精细化学品的生产环境 资源效率得到提高. 总之, 最近的均相催化氢化 $\mathrm{CO}_{2}$ 作 为 $\mathrm{C}_{1}$ 合成子取得了一些开创性的研究和突破进展, 已 经证明了利用新型金属有机催化剂以 $\mathrm{CO}_{2} / \mathrm{H}_{2}$ 的组合作 为 $\mathrm{C}_{1}$ 合成子进行反应的应用前景. 因此金属有机络合 物催化的氢化 $\mathrm{CO}_{2}$ 合成甲酸、盐及衍生物，甲醛，甲醇 及作为 $\mathrm{C}_{1}$ 合成子合成一些高附加值精细化学品的研究, 通过对配体的设计、合成及反应机理的研究，开发一些 新的 $\mathrm{CO}_{2}$ 氢化转化方法, 一方面可以促进能源系统中可 再生能源氢气向化学产品的转化, 另一方面也减少了当 今石油化工工业中的化学生产对环境的影响. 因此, 通 过化学家和化学工程师跨学科的研究可以为能源利用 和精细化学品生产的可持续发展做出重要贡献.

\section{Referenes}

[1] (a) Thomas, J. M.; Harris, K. D. M. Energy Environ. Sci. 2016, 9 , 687.

(b) Klankermayer, J.; Wesselbaum, S.; Beydoun, K.; Leitner, W. Angew. Chem., Int. Ed. 2016, 55, 7296.

[2] Otto, A.; Grube, T.; Schiebahna, S.; Stoltenab, D. Energy Environ. Sci. 2015, 8, 3283.

[3] (a) Aresta, M.; Dibenedetto, A.; Angelini, A. Chem. Rev. 2014, 114, 1709 . 
(b) Metcalfe, I. S.; North, M.; Pasquale, R.; Thursfield, A. Energy Environ. Sci. 2010, 3, 212.

(c) North, M.; Wang, B.; Young, C. Energy Environ. Sci. 2011, 4, 4163 .

(d) Chapman, A. M.; Keyworth, C.; Kember, M. R.; Lennox, A. J. J.; Williams, C. K. ACS Catal. 2015, 5, 1581

(e) Darensbourg, D. J.; Wilson, S. J. Green Chem. 2012, 14, 2665.

(f) Ellis, W. C.; Jung, Y.; Mulzer, M.; Di Girolamo, R.; Lobkovsky, E. B.; Coates, G. W. Chem. Sci. 2014, 5, 4004.

(g) Martnez, J.; Castro-Osma, J. A.; Earlam, A.; Alonso-Moreno, C.; Otero, A.; Lara-Snchez, A.; North, M.; Rodrguez-Diguez, A. Chem.-Eur. J. 2015, 21, 9850.

(h) Comerford, J. W.; Ingram, I. D. V.; North, M.; Wu, X. Green Chem. 2015, 17, 1966.

(i) Martn, C.; Fiorani, G.; Kleij, A. W. ACS Catal. 2015, 5, 1353.

(j) Buonerba, A.; De Nisi, A.; Grassi, A.; Milione, S.; Capacchione,

C.; Vagin, S.; Rieger, B. Catal. Sci. Technol. 2015, 5, 118.

(k) Mikkelsen, M.; Jørgensen, M.; Krebs, F. C. Energy Environ. Sci. 2010, 3, 43 .

[4] (a) Bitter, J. H.; Seshan, K.; Lercher, J. A. J. Catal. 1997, 171, 279. (b) Gomes, C. D. N.; Jacquet, O.; Villiers, C.; Thury, P.; Ephritikhine, M.; Cantat, T. Angew. Chem., Int. Ed. 2012, 51, 187.

[5] Li, Y.-N.; Ma, R.; He, L,-N.; Diao, Z.-F. Catal. Sci. Technol. 2014, $4,1498$.

[6] Reutemann, W.; Kieczka H. In UllmannÏs Encyclopedia of Industrial Chemistry, Wiley-VCH, Weinheim, 2000.

[7] Farlow, M. W.; Adkins, H. J. Am. Chem. Soc. 1935, 57, 2222.

[8] Hao, C.-Y.; Wang, S.-P.; Li, M.-S.; Kang, L.-Q.; Ma, X.-B. Catal. Today 2011, 160, 184.

[9] (a) Jessop, P. G.; Ikariya, T.; Noyori, R. Chem. Rev. 1995, 95, 259. (b) Leitner, W. Angew. Chem., Int. Ed. Engl. 1995, 34, 2207.

(c) Jessop, P. G.; Joo, F.; Tai, C.-C. Coord. Chem. Rev. 2004, 248, 2425 .

(d) Federsel, C.; Jackstell, R.; Beller, M. Angew. Chem., Int. Ed. 2010, 49, 6254 .

[10] Inoue, Y.; Izumida, H.; Sasaki, Y.; Hashimoto, H. Chem. Lett. 1976, 863.

[11] Graf, E.; Leitner, W. J. Chem. Soc., Chem. Commun. 1992, 4.

[12] Gassner, F.; Leitner, W. J. Chem. Soc., Chem. Commun. 1993, 1465.

[13] Jessop, P. G.; Ikariya, T.; Noyori, R. Nature 1994, 368, 231.

[14] (a) Himeda, Y.; Onozawa-Komatsuzaki, N.; Sugihara, H.; Arakawa, H.; Kasuga, K. Organometallics 2004, 23, 1480.

(b) Himeda, Y.; Onozawa-Komatsuzaki, N.; Sugihara, H.; Kasuga, K. Organometallics 2007, 26, 702.

[15] Tanaka, R.; Yamashita, M.; Nozaki, K. J. Am. Chem. Soc. 2009, 131,14168

[16] Azua, A.; Sanz, S.; Peris, E. Chem.-Eur. J. 2011, 17, 3963.

[17] (a) Filonenko, G. A.; Putten, R. V.; Schulpen, E. N.; Hensen, E. J. M.; Pidko, E. A. Chem CatChem 2014, 6, 1526.

(b) Filonenko, G. A.; Conley, M. P.; Copéret, C.; Lutz, M.; Hensen, E. J. M.; Pidko, E. A. ACS Catal. 2013, 3, 2522.

[18] Tai, C.-C.; Chang, T.; Roller, B.; Jessop, P. G. Inorg. Chem. 2003, 42,7340 .

[19] (a) Federsel, C.; Boddien, A.; Jackstell, R.; Jennerjahn, R.; Dyson, P. J.; Scopelliti, R.; Laurenczy, G.; Beller, M. Angew. Chem., Int. Ed. 2010, 49, 9777.

(b) Ziebart, C.; Federsel, C.; Anbarasan, P.; Jackstell, R.; Baumann, W.; Spannenberg, A.; Beller, M. J. Am. Chem. Soc. 2012, 134, 20701.

[20] Langer, R.; Diskin-Posner, Y.; Leitus, G.; Shimon, L. J. W.; Ben-David, Y.; Milstein, D. Angew. Chem., Int. Ed. 2011, 50, 9948.

[21] Federsel, C.; Ziebart, C.; Jackstell, R.; Baumann, W.; Beller, M. Chem.-Eur. J. 2012, 18, 72.

[22] Badiei, Y. M.; Wang, W.-H.; Hull, J. F.; Szalda, D. J.; Muckerman, J. T.; Himeda, Y.; Fujita, E. Inorg. Chem. 2013, 52, 12576.

[23] Jeletic, M. S.; Mock, M. T.; Appel, A. M.; Linehan, J. C. J. Am. Chem. Soc. 2013, 135, 11533.

[24] Taqui Khan, M. M.; Halligudi, S. B.; Rao, N. N.; Shukla, S. J. Mol.
Catal. 1989, 51, 161

[25] Hayashi, H.; Ogo, S.; Fukuzumi, S. Chem. Commun. 2004, 2714.

[26] Zhao, G.; Joó, F. Catal. Commun. 2011, 14, 74.

[27] Moret, S.; Dyson, P. J.; Laurenczy, G. Nat. Commun. 2014, 5, 4017

[28] Tsai, J. C.; Nicholas, M. J. Am. Chem. Soc. 1992, 114, 5117;

[29] Wesselbaum, S.; Hintermair, U.; Leitner, W. Angew. Chem., Int. Ed. 2012, 51,8585 .

[30] Mondal, B.; Neese, F.; Ye, S.-F. Inorg. Chem. 2015, 54, 7192.

[31] (a) Zhang, Y.-P.; Fei, J.-H.; Yu, Y.-M.; Zheng, X.-M. Catal. Lett. 2004, 93, 231.

(b) Zhang, Y.-P.; Fei, J.-H.; Yu, Y.-M.; Zheng, X.-M. Catal. Commun. 2004, 5, 643 .

[32] Yu, Y.-M.; Zhang, Y.-P.; Fei, J.-H.; Zheng, X.-M. Chin. J. Chem. 2005, 23, 977.

[33] Yu, Y.-M.; Fei, J.-H.; Zhang, Y.-P.; Zheng, X.-M. Chin. Chem. Lett. 2006, 17, 1097.

[34] Xu, Z.; McNamara, N. D.; Neumann, G. T.; Schneider, W. F.; Hicks, J. C. Chem CatChem 2013, 5, 1769.

[35] Arpe, H.-J. Industrial Organic Chemistry, Vol. 5, Wiley-VCH, Weinheim, 2010

[36] Jessop, P. G.; Hsiao, Y.; Ikariya, T.; Noyori, R. J. Chem. Soc., Chem. Commun. 1995, 707.

[37] Krçcher, O.; Kçppel, R. A.; Baiker, A. Chem. Commun. 1997, 453.

[38] Yadav, M.; Linehan, J. C.; Karkamkar, A. J.; Eide, E. V. D.; Heldebrant, D. J. Inorg. Chem. 2014, 53, 9849.

[39] Evans, G. O.; Newell, C. J. Inorg. Chim. Acta 1978, 31, L387.

[40] Federsel, C.; Boddien, A.; Jackstell, R.; Jennerjahn, R.; Dyson, P. J.; Scopelliti, R.; Laurenczy, G.; Beller, M. Angew. Chem., Int. Ed. 2010, 49, 9777.

[41] Darensbourg, D. J.; Ovalles, C. J. Am. Chem. Soc. 1984, 106, 3750.

[42] Chen, Y.- Z.; Lau, C. P. J. Mol. Catal. A 1995, 101, 33.

[43] Huff, C. A.; Sanford, M. S. J. Am. Chem. Soc. 2011, 133, 18122

[44] Jessop, P. G.; Ikariya, T.; Noyori, R. Chem. Rev. 1995, 95, 259.

[45] Jessop, P. G. In The Handbook of Homogeneous Hydrogenation Eds.: Vries, J. G. D.; Elsevier, C. J., Wiley-VCH, Weinheim, 2007, p. 489.

[46] Schmid, L.; Schneider, M. S.; Engel, D.; Baiker, A. Catal. Lett. 2003, 88, 105.

[47] Farlow, M. W.; Adkins, H. J. Am. Chem. Soc. 1935, 57, 2222.

[48] Liu, J.-L.; Guo. C.-K.; Zhang, Z.-F.; Jiang, T.; Liu, H.-Z.; Song. J.-L.; Fan, H.-L.; Han, B.-X. Chem. Commun. 2010, 46, 5770.

[49] Schaub, T.; Paciello, R.; Pazicky, M.; Fachinetti, G.; Preti, D. WO 2013014160, 2013 [Chem. Abstr. 2013, 158, 218798].

[50] Schaub, T.; Paciello, R.; Pazicky, M.; Fachinetti, G.; Preti, D. US 20130102807, 2013 [Chem. Abstr. 2013, 158, 576492].

[51] Haynes, P.; Slaugh, L. H.; Kohnle, J. F. Tetrahedron Lett. 1970, 365.

[52] Jessop, P. G.; Hsiao, Y.; Ikariya, T.; Noyori, R. J. Am. Chem. Soc. 1994, 116, 8851

[53] Krçcher, O.; Kçppel, R. A.; Baiker, A. Chem. Commun. 1997, 453.

[54] Zhang, L.; Han, Z.; Zhao, X.; Wang, Z.; Ding, K. Angew. Chem., Int. Ed. 2015, 54, 6186.

[55] Reuss, G.; Disteldorf, W.; Gamer, A. A. O. Hilt in Ullmanňs Encyclopedia of Industrial Chemistry, Wiley-VCH, Weinheim, 2000.

[56] Wang, Z.-C.; Dietl, N.; Kretschmer, R.; Ma, J.-B.; Weiske, T.; Schlangen, M.; Schwarz, H. Angew. Chem., Int. Ed. 2012, 51, 3703.

[57] Lee, D.-K.; Kim, D.-S.; Kim, S.-W. Appl. Organomet. Chem. 2001, $15,148$.

[58] Gambarotta, S.; Strologo, S.; Floriani, C.; Chiesi-Villa, A.; Guastini, C. J. Am. Chem. Soc. 1985, 107, 6278

[59] (a) Tso, C. C.; Cutler, A. R. J. Am. Chem. Soc. 1986, 108, 6069. (b) Steffey, B. D.; Vites, J. C.; Cutler, A. R.; Organometallics 1991, 10, 3432 .

[60] Bontemps, S.; Vendier, L.; Sabo-Etienne, S. J. Am. Chem. Soc. 2014, 136, 4419

[61] Heim, L. E.; Schlorer, N. E.; Choi, J. H.; Prechtl, M. H. Nat. Commun. 2014, 5, 3621.

[62] Bertau, M.; Offermanns, H.; Plass, L.; Schmidt, F.; Wernicke, H.-J. 
Methanol: The Basic Chemical and Energy Feedstock of the Future Springer, Amsterdam, 2013.

[63] Arpe, H.-J. Industrial Organic Chemistry, Vol. 5, Wiley-VCH, Weinheim, 2010.

[64] Baerns, M.; Behr, A.; Brehm, A.; Gmehling, J.; Hofmann, H.; Onken, U.; Renken, A. Technische Chemie, Wiley-VCH, Weinheim, 2006.

[65] Asinger, F. Methanol-Chemie- und Energierohstoff, Springer, Heidelberg, 1986 (in German)

[66] (a) Olah, G. A. Angew. Chem., Int. Ed. 2005, 44, 2636.

(b) Olah, G. A.; Goeppert, A.; Prakash, G. K. S. Beyond Oil and Gas: The Methanol Economy, Wiley-VCH, Weinheim, 2006.

[67] Markewitz, P.; Kuckshinrichs, W.; Leitner, W.; Linssen, J.; Zapp, P.; Bongartz, R.; Schreiber, A.; Muller, T. E. Energy Environ. Sci. 2012, 5, 7281.

[68] Davies, P.; Snowdon, F.; Bridger, G. W.; Hughes, D. O.; Young, P. W. DE 1241429, 1963.

[69] Lim, H.-W.; Park, M.-J.; Kang, S.-H.; Chae, H.-J.; Bae, J. W.; Jun, K.-W. Ind. Eng. Chem. Res. 2009, 48, 10448.

[70] Saito, M.; Fujitani, T.; Takeuchi, M.; Watanabe, T. Appl. Catal. A 1996, 138,311 .

[71] Koenig, P.; Koenig, P. D. US 5631302, 1997 [Chem. Abstr. 1995, 124, 33563].

[72] Behrens, M.; Stud, F.; Kasatkin, I.; Kühl, S.; Hävecker, M.; Abild-Pedersen, F.; Zande, S.; Girgsdies, F.; Kurr, P.; Kniep, B.-L.; Tovar, M.; Fischer, R. W.; Nørskov, J. K.; Schlögl, R. Science 2012, $336,893$.

[73] Studt, F.; Behrens, M.; Kunkes, E. L.; Thomas, N.; Zander, S.; Tarasov, A.; Schuman, J.; Frei, E.; Varley, J. B.; AbildPedersen, F.; Nørskov, J. K.; Schlögl, R. ChemCatChem 2015, 7, 1105.

[74] Tominaga, K-I.; Sasaki, Y.; Kawai, M.; Watanabe, T.; Saito M. J. Chem. Soc., Chem. Commun. 1993, 629.

[75] Balaraman, E.; Gunanathan, C.; Zhang, J.; Shimon, L. J. W.; Milstein, D. Nat. Chem. 2011, 3, 609.

[76] Balaraman, E.; Ben-David, Y.; Milstein, D. Angew. Chem., Int. Ed. 2011, 50, 11702.

[77] Balaraman, E.; Gnanaprakasam, B.; Shimon, L. J. W.; Milstein, D. J. Am. Chem. Soc. 2010, 132, 16756.

[78] Han, Z.-B.; Rong, L.-C.; Wu, J.; Zhang, L.; Wang, Z.; Ding, K.-L.; Angew. Chem., Int. Ed. 2012, 51, 13041.

[79] Rezayee, N. M.; Huff, C. A.; Sanford, M. S. J. Am. Chem. Soc. 2015, 137, 1028.

[80] Kothandaraman, J.; Goeppert, A.; Czaun, M.; Olah, G. A.; Prakash, G. K. J. Am. Chem. Soc. 2016, 138, 778.

[81] Wesselbaum, S.; Stein, T. v.; Klankermayer, J.; Leitner, W. Angew. Chem., Int. Ed. 2012, 51, 7499.

[82] (a) Teunissen, H. T.; Elsevier, C. J. Chem. Commun. 1997, 667. (b) Teunissen, H. T. Chem. Commun. 1998, 1367. (c) Magro, A. A. N.; Eastham, G. R.; Cole-Hamilton, D. J. Chem. Commun. 2007, 3154.

(d) Rosi, L.; Frediani, M.; Frediani, P. J. Organomet. Chem. 2010 $695,1314$.

(e) Geilen, F. M. A.; Engendahl, B.; Harwardt, A.; Marquardt, W.; Klankermayer, J.; Leitner, W. Angew. Chem., Int. Ed. 2010, 49, 5510 .

(f) Coetzee, J.; Dodds, D. L.; Klankermayer, J.; Brosinski, S.; Leitner, W.; Slawin, A. M. Z.; Cole-Hamilton, D. J. Chem.-Eur. J. 2013, 19, 11039 .

(g) Stein, T. V.; Weigand, T.; Merkens, C.; Klankermayer, J.; Leitner, W. Chem CatChem. 2013, 5, 439.

(h) Stein, T. V.; Meuresch, M.; Limper, D.; Schmitz, M.; Hölscher, M.; Coetzee, J.; Cole-Hamilton, D. J.; Klankermayer, J.; Leitner, W. J. Am. Chem. Soc. 2014, 136, 13217.

[83] Zhang, L.-L.; Han, Z.-B.; Zhang, L.; Li, M.-X.; Ding, K.-L. Chin. J. Org. Chem. 2016, 36, 1824 (in Chinese).

(张琳莉, 韩召斌, 张磊, 李明星, 丁奎岭, 有机化学 2016, 36, 1824.)

[84] Wesselbaum, S.; Moha, V.; Meuresch, M.; Brosinski, S.; Thenert, K. M.; Kothe, J.; Stein, T. V.; Englert, U.; Hölscher, M.; Klankermayer, J.; Leitner, W. Chem. Sci. 2015, 6, 693.

[85] Ren, X.-Y.; Zheng, Z.-Y.; Zhang, L.; Wang, Z.; Xia, C.-G.; Ding, K.-L. Angew. Chem., Int. Ed. 2017, 56, 310.

[86] Schneidewind, J.; Adam, R.; Baumann, W.; Jackstell, R.; Beller, M. Angew. Chem., Int. Ed. 2017, 56, 1890.

[87] Tominaga, K-I.; Sasaki, Y. Catal. Commun. 2000, $1,1$.

[88] Liu, Q.; Wu, L.-P.; Fleischer, I.; Selent, D.; Franke, R.; Jackstell, R.; Beller, M. Chem. Eur. J. 2014, 20, 6888.

[89] Ali, M.; Gual, A.; Ebeling, G.; Dupont, J. ChemCatChem 2014, 6, 2224.

[90] Srivastava, V. K.; Eilbracht, P. Catal. Commun. 2009, 10, 1791.

[91] Ostapowicz, T. G.; Schmitz, M.; Krystof, M.; Klankermayer, J.; Leitner, W. Angew. Chem., Int. Ed. 2013, 52, 12119.

[92] Wu, L.; Liu, Q.; Fleischer, I.; Jackstell, R.; Beller, M. Nat. Commun. 2014, 5, 3091.

[93] Fleischer, I.; Gehrtz, P. H.; Hirschbeck, V. Chem. Commun. 2015, $51,12574$.

[94] Klankermayer, J.; Leitner, W.; Beydoun, K.; Stein, T. V. Angew. Chem., Int. Ed. 2013, 52, 9554.

[95] Li, Y.; Sorribes, I.; Yan, T.; Junge, K.; Beller, M. Angew. Chem., Int. Ed. 2013, 52, 12156.

[96] Klankermayer, J.; Leitner, W.; Beydoun, K.; Stein, T. V. Angew. Chem., Int. Ed. 2013, 52, 9554.

[97] Beydoun, K.; Ghattas, G.; Thenert, K.; Klankermayer, J.; Leitner, W. Angew. Chem., Int. Ed. 2014, 53, 11010.

[98] Li, Y.; Sorribes, I.; Yan, T.; Junge, K.; Beller, M. Angew. Chem., Int. Ed. 2013, 52, 12156. 